\title{
Localization and mass spectra of various matter fields on Weyl thin brane
}

\author{
Tao-Tao Sui ${ }^{1, \mathrm{a}}$, Li Zhao ${ }^{1, \mathrm{~b}}$, Yu-Peng Zhang ${ }^{1, \mathrm{c}}$, Qun-Ying Xie ${ }^{2, \mathrm{~d}}$ \\ ${ }^{1}$ Institute of Theoretical Physics, Lanzhou University, Lanzhou 730000, People's Republic of China \\ ${ }^{2}$ School of Information Science and Engineering, Lanzhou University, Lanzhou 730000, People's Republic of China
}

Received: 21 January 2017 / Accepted: 14 May 2017 / Published online: 19 June 2017

(C) The Author(s) 2017. This article is an open access publication

\begin{abstract}
It has been shown that the thin brane model in a five-dimensional Weyl gravity can deal with the wrongsigned Friedmann-like equation in the Randall-Sundrum-1 (RS1) model. In the Weyl brane model, there are also two branes with opposite brane tensions, but the four-dimensional graviton (the gravity zero mode) is localized near the negative tension brane, while our four-dimensional universe is localized on the positive tension brane. In this paper, we consider the mass spectra of various bulk matter fields (i.e., scalar, vector, and fermion fields) on the Weyl brane. It is shown that the zero modes of those matter fields can be localized on the positive tension brane under some conditions. The mass spectra of the bulk matter fields are equidistant for the higher excited states, and relatively sparse for the lower excited states. The size of the extra dimension determines the gap of the mass spectra. We also consider the correction to the Newtonian potential in this model and it is proportional to $1 / r^{3}$.
\end{abstract}

\section{Introduction}

Over the last decades, there was a great interest to construct a unified theory in higher-dimensional spacetime. The higherdimensional theory dates back to the work of Kaluza and Klein $[1,2]$ in the 1920 s. They tried to unify electromagnetism with gravity by assuming that the $g_{5 \mu}$ component of the five-dimensional metric represents the electromagnetic potential $[1,2]$. Subsequently, the brane world theories appear as an alternative theory to solve the gauge hierarchy and cosmological constant problems (see e.g., Refs. [3-6]) with a fundamental idea that the visible universe is localized on a 3-brane embedded in a higher-dimensional bulk.

\footnotetext{
a e-mail: suitt14@1zu.edu.cn

b e-mail: lizhao@1zu.edu.cn

c e-mail: zhangyupeng14@1zu.edu.cn

de-mail: xieqy@1zu.edu.cn
}

Most research as regards brane worlds is based on a Riemann geometry [7-15]. The non-Riemannian models can be classified within the language of metric-affine gravity [1619]. Generally, there are two types of generalized Riemann geometries, Riemann-Cartan geometry and Weyl geometry [17]. Cartan introduced the torsion $T$ to represent the antisymmetric piece of the connection, which may be related to spin. When the connection includes a geometric scalar $\omega$, there will be a nonmetricity part called $Q$, which is related to the scalar $\omega$. In Riemann-Cartan geometry, the torsion $T \neq 0$, and the nonmetricity part $Q=0$. When $T=0$, the Riemann-Cartan geometry can degenerate into Riemann geometry. The Weyl geometry is an affine manifold described by a pair $\left(g_{M N}, \omega_{M}\right)$, where $g_{M N}$ is the metric and $\omega_{M}$ is the "gauge" vector as the gradient of a scalar function $\omega$. In the Weyl geometry, $T=0$ and $Q \neq 0$. When $Q=0$, the Weyl geometry can also degenerate into Riemann geometry. As a generalization of Riemann, Weyl geometry has received much attention in the studies in field theory and cosmology [20-23]. Moreover, in Refs. [16,20,25-29], the authors have studied the Weyl brane world scenario and obtained various solutions of Minkowski thick branes.

In the brane world scenario with all matter fields (scalar, vector, and fermion fields) propagating in the bulk, the zero modes of these bulk fields, i.e., the four-dimensional matter fields in the Standard Model, should be localized on the brane complying with the present observations. So, localization of various bulk matter fields on a brane is an important and interesting issue. References $[7,8,30]$ showed that the zero mode of a free massless scalar field can be localized on the RS brane or its generalized branes. In order to ensure that the zero mode of vector field can be localized on the brane, the case of generalized RS branes with more extra dimensions was considered [31,32], a dynamical mass term was included in gauge field localization [33,34], and in Refs. [35-44] the authors introduced the interaction with the background scalar field. Localization of the fermion zero mode on 
a brane is especially important. It has been demonstrated that localization mechanisms, e.g. Yukawa coupling $\eta \bar{\Psi} F(\phi) \Psi$ [11-13,16,45-52,52-55], derivative scalar-fermion coupling $\eta \bar{\Psi} \Gamma^{M} \partial_{M} F(\phi) \gamma^{5} \Psi$ [56], and derivative geometryfermion coupling $\eta \bar{\Psi} \Gamma^{M} \partial_{M} F(R) \gamma^{5} \Psi$ [57] can ensure that the fermion zero mode can be localized on the brane.

In the RS1 model [4], the authors assumed that there are two thin branes located at the boundaries of the extra dimension. One of them is the negative tension brane (called Tev brane, where our universe is resided), and the other is the positive tension brane (called the Planck brane, where the spin-2 gravitons are localized). The warping of the extra dimension in RS1 model can solve the famous gauge hierarchy problem, but the fundamental assumption that our universe is localized on the negative tension brane can lead to a wrong-signed Friedmann-like equation [58-60]. The authors of Ref. [61] presented a generalized RS1 model in the Weyl integrable geometry, which can give a correct Friedmann-like equation since our universe is assumed to be located on the positive tension brane. It was shown that the brane system is stable under the tensor perturbation and the gravity zero mode is localized near the negative tension brane, whereas the matter fields should be confined on the positive tension brane, since our universe is located on this brane.

In this paper, we will do further research on the localization of various bulk matter fields in this Weyl brane model and check whether the zero modes of these fields can be confined on the positive tension brane. We will consider a dilaton coupling between the bulk matter fields and the background scalar field. It will be found that the coupling is necessary for most of the bulk fields. For the fermion case, we consider the usual Yukawa coupling $\eta \bar{\Psi} F(\omega) \Psi$ to obtain analytical wave functions of the fermion KK modes. This paper is organized as follows. In Sect. 2, we give a brief review of the Weyl brane model proposed in Ref. [61]. In Sect. 3, we investigate localization and mass spectra of the scalar, vector, and fermion fields on the Weyl brane. In Sect. 4, gravitational fluctuations and a correction to the Newtonian potential are discussed. Finally, we give a brief conclusion and summary in Sect. 5.

\section{Review of Weyl brane model}

The brane model we consider here is based on the fivedimensional Weyl geometric action where the gravity is nonminimally coupled with a background scalar field $\omega$. The action is given by [61]

$$
S_{5}=\frac{M_{*}^{3}}{2} \int_{M_{5}^{W}} \mathrm{~d}^{5} x \sqrt{-g} e^{k \omega}\left[R-(4+4 k) \nabla_{K} \omega \nabla^{K} \omega\right] .
$$

Here $R$ is the five-dimensional Weyl Ricci scalar, $M_{*}^{-3} \equiv$ $8 \pi G_{5}$ is the fundamental scale of gravity, the Latin letters $M, N, K=0,1,2,3,5$, and $\omega_{, K}$ is a gradient of the Weyl scalar $\omega$. In what follows it is convenient to denote the physical four coordinates by $x^{\mu}$, and the extra dimension by $y$.

In this frame the Weyl Ricci tensors are constructed from $\Gamma_{L N}^{M}$ in a standard way,

$R_{L K N}^{M}=\partial_{K} \Gamma_{L N}^{M}-\partial_{N} \Gamma_{L K}^{M}+\Gamma_{K P}^{M} \Gamma_{N L}^{P}-\Gamma_{N P}^{M} \Gamma_{K L}^{P}$,

$R_{L N}=R_{L M N}^{M}$,

$R=R_{5}=R_{L}^{L}$,

where the Weyl affine connection is expressed as

$\Gamma_{M N}^{P}=\left\{M_{M N}^{P}\right\}-\frac{1}{2}\left(\delta_{N}^{P} \nabla_{M} \omega+\delta_{M}^{P} \nabla_{N} \omega-g_{M N} \nabla_{P} \omega\right)$,

with $\left\{\begin{array}{l}P \\ M N\end{array}\right\}$ representing the Riemannian Christoffel symbol. Now with some algebra, the Weyl Ricci tensor $R_{M N}$ can be separated into a Riemannian Ricci tensor part $\hat{R}_{M N}$ plus some other terms with respect to the Weyl scalar function, i.e.,

$$
\begin{aligned}
R_{M N}= & \hat{R}_{M N}+\frac{3}{2} \nabla_{M} \nabla_{N} \omega+\frac{1}{2} g_{M N} \nabla_{P} \nabla^{P} \omega \\
& +\frac{3}{4}\left(\nabla_{M} \omega \nabla_{N} \omega-g_{M N} \nabla_{K} \omega \nabla^{K} \omega\right),
\end{aligned}
$$

where the hatted magnitudes and operators are defined by the Christoffel symbol. Then from the action (1), the equations of motion are

$$
\begin{aligned}
\hat{G}_{M N}= & (4+4 k)\left(\nabla_{M} \omega \nabla_{N} \omega-\frac{1}{2} g_{M N} \nabla^{K} \omega \nabla_{K} \omega\right) \\
& +k\left(\nabla_{M} \nabla_{N} \omega-g_{M N} \nabla^{K} \nabla_{K} \omega\right) \\
& +k^{2}\left(\nabla_{M} \nabla_{N} \omega-g_{M N} \nabla^{K} \omega \nabla_{K} \omega\right), \\
k \hat{R}= & -(8+8 k) \nabla^{K} \nabla_{K} \omega-4\left(k+k^{2}\right) \nabla^{K} \omega \nabla_{K} \omega .
\end{aligned}
$$

Further, we can write the field equation (5) in the form of the Einstein equation by moving all Weyl scalar terms to the right-hand side to compose an effective energy-momentum tensor:

$\hat{G}_{M N}=\hat{T}_{M N}$.

The authors in Refs. [20,24,25,62-65] studied thick brane solutions, by considering the metric that satisfies fourdimensional Poincaré invariance. The localization and mass spectrum problems of bulk matter fields on the Weyl Minkowski thick branes were discussed in Refs. [16,20, 24-29]. We are interested in the Weyl Minkowski thin branes with space $S^{1} / Z_{2}$, for which the metric with fourdimensional Lorentz invariance in a five-dimensional Weyl spacetime is given by 


$$
\begin{aligned}
\mathrm{d} s^{2} & =a^{2}(y) \eta_{\mu \nu} \mathrm{d} x^{\mu} \mathrm{d} x^{\nu}+d y^{2} \\
& =a^{2}(z)\left(\eta_{\mu \nu} \mathrm{d} x^{\mu} \mathrm{d} x^{\nu}+\mathrm{d} z^{2}\right),
\end{aligned}
$$

where $z$ is the conformal coordinate of the extra dimension with $z \in\left[-z_{b}, z_{b}\right]$ and it relates to the physical coordinate $y$ by a coordinate transformation $\mathrm{d} y=a(z) \mathrm{d} z$. One assumes that the background scalar field $\omega$ only depends on the extra dimension.

According to the metric (8), the field equations in the bulk can be expressed in terms of the warp factor $a$ and the scalar field $\omega$, as in the following set of equations:

$k \omega^{\prime \prime}+\left(k^{2}+2 k+2\right) \omega^{\prime 2}+2 k \frac{a^{\prime}}{a} \omega^{\prime}+3 \frac{a^{\prime \prime}}{a}=0$,

$(1+k) \omega^{2}-2 k \frac{a^{\prime}}{a} \omega^{\prime}-3 \frac{a^{\prime 2}}{a^{2}}=0$,

$2(1+k)\left(\omega^{\prime \prime}+3 \frac{a^{\prime}}{a} \omega^{\prime}\right)+k(1+k) \omega^{\prime 2}-k\left(\frac{a^{\prime 2}}{a^{2}}+2 \frac{a^{\prime \prime}}{a}\right)=0$,

where the prime denotes the derivative with respect to $z$. The above field equations admit the following brane solution [61]:

$$
\begin{aligned}
& a(z)=(1+\beta|z|)^{\frac{1}{3}\left(1+\frac{k}{\alpha}\right)}, \\
& \omega(z)=-\frac{1}{\alpha} \ln (1+\beta|z|),
\end{aligned}
$$

where $\alpha=\sqrt{k^{2}+3 k+3}$, the parameter $\beta>0$ and $k<-1$.

The five-dimensional effective energy-momentum tensor is given as usual by

$\hat{T}_{M N}=\frac{2}{\sqrt{-g}} \frac{\delta \hat{S}}{\delta g_{M N}}=\hat{R}_{M N}-\frac{1}{2} g_{M N} \hat{R}$,

with $\hat{S}$ the effective matter action. In this case the effective energy density is given by the null-null component $\hat{T}_{0}^{0}$ of the energy-momentum tensor,

$\rho(z)=\frac{\beta^{2}}{3 \alpha^{2}} \frac{\left(k^{2}+\alpha k+6 k+6\right)}{(1+z \beta)^{\frac{2}{3}\left(4+\frac{k}{\alpha}\right)}}-\frac{2 k \beta\left[\delta(z)-\delta\left(z-z_{b}\right)\right]}{\alpha(1+\beta|z|)^{\frac{5}{2}+\frac{2 k}{3 \alpha}}}$.

This expression directly shows that there are two delta functions appearing at each boundary, so they stand for two thin branes, namely a positive tension brane at the origin and a negative one at the boundary $z_{b}$. These tensions compensate for the effects produced by the bulk component and hence ensure the existence of four-dimensional flat branes. So the brane configuration of this case is similar to that of the RS1 model. However, localization of massless graviton in these two brane scenarios will be quite different, and in Ref. [61] one can suppose that the standard model fields are confined on the positive tension brane at $z=0$, so this is crucial to overcome the severe cosmological problem of the RS1 model.

\section{Localization of various matter fields}

In this section, we investigate the localization and mass spectra of various bulk matter fields, including the spin-0 scalar, spin- 1 vector and spin-1/2 fermion fields on the Weyl brane. A natural mechanism for localizing spin-0 scalar and spin1 vector fields on the brane involves the couplings with the background scalar field. For the case of spin-1/2 fermions, we will investigate the usual Yukawa coupling $\eta \bar{\Psi} F(\omega) \Psi$.

\subsection{Spin-0 scalar field}

Firstly, we explore the localization condition and the mass spectrum for a massless scalar field on the Weyl brane by considering two types of coupling with the background scalar fields, dilaton coupling and Higgs potential coupling [14].

\subsubsection{The dilaton coupling}

Let us consider a massless scalar field $\Phi$ coupled with the dilaton field $\omega$ via the following five-dimensional action:

$S_{\mathrm{s}}=-\frac{1}{2} \int \mathrm{d}^{5} x \sqrt{-g} e^{\lambda \omega} g^{M N} \partial_{M} \Phi \partial_{N} \Phi$,

where $\lambda$ is a dimensionless coupling constant. For convenience, we rewrite the warp factor so that the metric (8) takes the form

$$
\begin{aligned}
\mathrm{d} s^{2} & =a^{2}(z)\left(\eta_{\mu \nu} \mathrm{d} x^{\mu} \mathrm{d} x^{\nu}+\mathrm{d} z^{2}\right) \\
& =e^{2 A(z)}\left(\eta_{\mu \nu} \mathrm{d} x^{\mu} \mathrm{d} x^{\nu}+\mathrm{d} z^{2}\right) .
\end{aligned}
$$

Following (13) and (14), the equation of motion for the scalar field satisfies the five-dimensional Klein-Gordon equation,

$\partial_{P}\left(\sqrt{-g} \partial^{P} \Phi\right)=0$.

Making the KK decomposition

$\Phi\left(x^{\mu}, z\right)=\sum_{n} \phi_{n}\left(x^{\mu}\right) \chi_{n}(z) e^{-\varrho A(z)}$,

with the quantity $\varrho=\frac{3}{2} \frac{k+\alpha-\lambda}{k+\alpha}$, and demanding that $\phi_{n}\left(x^{\mu}\right)$ satisfies the four-dimensional massive Klein-Gordon equation $\left(\partial_{\mu} \partial^{\mu}-m_{n}^{2}\right) \phi_{n}=0$, we obtain the equation of the extradimensional part $\chi_{n}(z)$ of the scalar KK mode, which can be converted into the following Schrödinger-like equation:

$\left[-\partial_{z}^{2}+V_{\mathrm{s}}(z)\right] \chi_{n}(z)=m_{n}^{2} \chi_{n}(z)$,

where $m_{n}$ is the mass of the scalar KK mode $\phi_{n}\left(x^{\mu}\right)$ and the effective potential $V_{\mathrm{S}}(z)$ reads

$V_{\mathrm{s}}(z)=\varrho \partial_{z}^{2} A(z)+\left[\varrho \partial_{z} A(z)\right]^{2}$.

It is clear that the effective potential $V_{\mathrm{s}}$ is only dependent on the warp factor. In fact, the Schrödinger-like equation 
(17) can be written as $\mathcal{H} \chi_{n}=m_{n}^{2} \chi_{n}$, where the Hamiltonian operator is given by $\mathcal{H}=Q^{+} Q$ with $Q=-\partial_{z}+\varrho \partial_{z} A$. Since the operator $\mathcal{H}$ is positive definite, there are no KK modes with negative $m_{n}^{2}$.

By substituting the KK decomposition (16) into the fivedimensional scalar action (13) and introducing the following orthonormality conditions:

$$
\int_{-z_{b}}^{+z_{b}} \chi_{m}(z) \chi_{n}(z) \mathrm{d} z=\delta_{m n}
$$

we get the effective low-energy theory in a four-dimensional familiar form

$S_{\mathrm{s}}=-\frac{1}{2} \sum_{n} \int \mathrm{d}^{4} x\left(\eta^{\mu \nu} \partial_{\mu} \phi_{n} \partial_{\nu} \phi_{n}+m_{n}^{2} \phi_{n}^{2}\right)$,

where we have used Eq. (17).

Due to the explicit forms of $\varrho$ and $A(z)$, the expression of the effective potential $V_{\mathrm{s}}(z)$ corresponding to Eq. (18) is given by

$$
\begin{aligned}
V_{\mathrm{s}}(z)= & \frac{\left[(k-\lambda)^{2}-\alpha^{2}\right] \beta^{2}}{4 \alpha^{2}(1+\beta|z|)^{2}} \\
& +s \beta\left[\delta(z)-\frac{1}{1+\beta z_{b}} \delta\left(z-z_{b}\right)\right],
\end{aligned}
$$

where $s=\frac{\alpha+k-\lambda}{\alpha}$. The value of $V_{\mathrm{s}}(z)$ at $z=0$ is

$V_{\mathrm{s}}(0)=\frac{\left[(k-\lambda)^{2}-\alpha^{2}\right] \beta^{2}}{4 \alpha^{2}}+s \beta \delta(0)$.

In order to localize the scalar zero mode on the positive tension brane, the effective potential $V_{\mathrm{s}}(z)$ should be negative at $z=0$, which results in the following condition:

$\lambda>k+\alpha$.

By setting $m=0$ in Eq. (17), we easily get the normalized zero mode of the scalar field for $\lambda \neq k+2 \alpha$ :

$\chi_{0}(z)=\sqrt{\frac{\beta(k+2 \alpha-\lambda)}{2 \alpha\left[\left(1+\beta z_{b}\right)^{\frac{(k+2 \alpha-\lambda)}{\alpha}}-1\right]}}(1+\beta|z|)^{\frac{k+\alpha-\lambda}{2 \alpha}}$,

which is indeed localized on the positive tension brane under the condition (23). When the extra dimension is infinite, the normalization condition $\int_{-\infty}^{+\infty} \chi_{0}(z)^{2} \mathrm{~d} z=1$ means that the localization condition for the scalar zero mode is much stronger: $\lambda>k+2 \alpha$. For $\lambda=k+2 \alpha$, the normalized scalar zero mode reads

$\chi_{0}(z)=\sqrt{\frac{\beta}{2 \ln \left(1+\beta z_{b}\right)}} \frac{1}{\sqrt{1+\beta|z|}} \quad(\lambda=k+2 \alpha)$,

and it is also localized on the positive tension brane. But if the extra dimension is infinite, the scalar zero mode is not normalized and hence we cannot get a localized scalar zero mode. When there is no coupling between the scalar and dilaton fields, i.e., the parameter $\lambda=0$, one can verify that the scalar zero mode is also localized on the positive tension brane when $z_{b}$ is finite.

In order to investigate the mass spectrum of the scalar KK modes, we redefine the following dimensionless parameters:

$|\bar{z}| \equiv \beta|z|, \quad \bar{m}_{n} \equiv \frac{m_{n}}{\beta}, \quad \bar{V}_{\mathrm{s}}(\bar{z}) \equiv \frac{V_{\mathrm{s}}(z)}{\beta^{2}}$,

with which the Schrödinger-like equation (17) is rewritten as $\left(-\partial_{\bar{z}}^{2}+\bar{V}_{\mathrm{s}}\right) \chi(\bar{z})=\bar{m}_{n}^{2} \chi(\bar{z})$,

where the effective potential $\bar{V}_{\mathrm{s}}$ reads

$\bar{V}_{\mathrm{s}}(z)=\frac{\left[(k-\lambda)^{2}-\alpha^{2}\right]}{4 \alpha^{2}(1+|\bar{z}|)^{2}}+c\left[\delta(\bar{z})-\frac{1}{1+\bar{z}_{b}} \delta\left(\bar{z}-\bar{z}_{b}\right)\right]$.

The general solution of Eq. (27) is given in terms of the combination of Bessel functions

$$
\begin{aligned}
& \chi_{n}(\bar{z})=N_{n}(1+|\bar{z}|)^{\frac{1}{2}} \\
& \quad \times\left[C_{1} \mathrm{~J}_{P_{\mathrm{s}}}\left(\bar{m}_{n}(|\bar{z}|+1)\right)+C_{2} \mathrm{Y}_{P_{\mathrm{s}}}\left(\bar{m}_{n}(|\bar{z}|+1)\right)\right],
\end{aligned}
$$

where $N_{n}$ is the normalization coefficient, $C_{1}$ and $C_{2}$ are the $m$-dependent parameters, $\mathrm{J}_{P}(z)$ and $\mathrm{Y}_{P}(z)$ are the Bessel functions of the first and second kinds.

Then we impose the two kinds of boundary condition (i.e., the Neumann boundary condition and the Dirichlet boundary condition) to calculate the mass spectra of scalar KK modes, respectively [66]. For the Neumann boundary condition $\left.\partial_{\bar{z}}\left(e^{-\varrho A(\bar{z})} \chi_{n}\right)\right|_{\bar{z}=0, \bar{z}=\bar{z}_{b}}=0$, we get the mass spectrum which is determined by the following condition:

$\mathcal{M}_{N}\left(\bar{m}_{n}\right) \equiv \mathrm{J}_{P_{\mathrm{Ns}}}\left(\bar{m}_{n}\left(\bar{z}_{b}+1\right)\right)+\mathcal{C}_{\mathrm{Ns}} \mathrm{Y}_{P_{\mathrm{Ns}}}\left(\bar{m}_{n}\left(\bar{z}_{b}+1\right)\right)=0$,

where $P_{\mathrm{Ns}}$ and $\mathcal{C}_{\mathrm{Ns}}$ are defined as

$P_{\mathrm{Ns}} \equiv \frac{\lambda-k}{2 \alpha}-1, \quad \mathcal{C}_{\mathrm{Ns}} \equiv-\frac{\mathrm{J}_{P_{\mathrm{Ns}}}\left(\bar{m}_{n}\right)}{\mathrm{Y}_{P_{\mathrm{Ns}}}\left(\bar{m}_{n}\right)}$.

For the Dirichlet boundary condition $e^{-\varrho A(\bar{z})} \chi_{n}=0$ at the boundaries $\bar{z}=0$ and $\bar{z}=\bar{z}_{b}$, we get the mass spectrum of the scalar KK modes which satisfies the following relation:

$\mathcal{M}_{D}\left(\bar{m}_{n}\right) \equiv \mathrm{J}_{P_{\mathrm{Ds}}}\left(\bar{m}_{n}\left(\bar{z}_{b}+1\right)\right)+\mathcal{C}_{\mathrm{Ds}} \mathrm{Y}_{P_{\mathrm{Ds}}}\left(\bar{m}_{n}\left(\bar{z}_{b}+1\right)\right)=0$,

where $P_{\mathrm{Ds}}$ and $\mathcal{C}_{\mathrm{Ds}}$ are given by

$P_{\mathrm{Ds}} \equiv \frac{\lambda-k}{2 \alpha}, \quad \mathcal{C}_{\mathrm{Ds}} \equiv-\frac{\mathrm{J}_{P_{\mathrm{Ds}}}\left(\bar{m}_{n}\right)}{\mathrm{Y}_{P_{\mathrm{Ds}}}\left(\bar{m}_{n}\right)}$.

From Eqs. (30) and (32), we see that the Neumann and Dirichlet boundary conditions give similar expressions which determine the mass spectrum, whereas the orders of the Bessel functions are different as shown in (31) and (33). By tuning the parameters $k$ and $\lambda$, the same orders of Bessel functions 


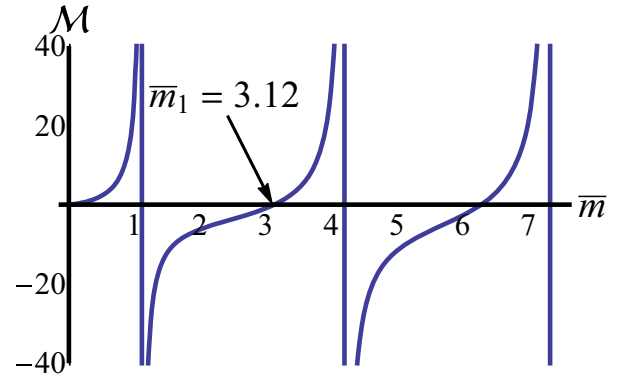

(a)

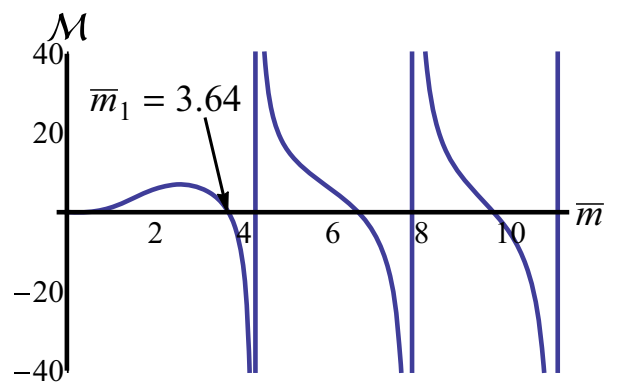

(b)

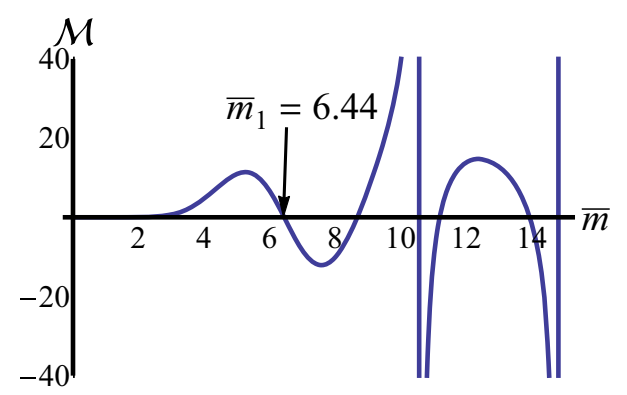

(c)

Fig. 1 The effect of the parameter $\lambda$ on the mass $\bar{m}_{1}$ of the first massive scalar KK mode. The other parameters are set to $k=-3$ and $\bar{z}_{b}=1$

can be obtained, and thus the expression of the mass spectrum derived by the Neumann boundary condition is equivalent to that derived by the Dirichlet boundary condition. Therefore, we only consider the Neumann boundary condition for later discussion.

According to Eq. (30), we plot the relation between $\mathcal{M}_{N}$ and $\bar{m}_{n}$ in Figs. 1, 2, 3, where the zero points represent the mass of the KK modes. Figure 1 shows that the effect of the parameter $\lambda$ on the mass $\bar{m}_{1}$ of the first massive scalar KK mode. It can be seen that the mass $\bar{m}_{1}$ increases with $\lambda$. On the other hand, there are some singular (vertical) lines in these figures, which come from the zero points of $\mathrm{Y}_{P_{\mathrm{Ns}}}\left(\bar{m}_{n}\right)$ in (31). Since the gaps between two arbitrary adjacent singular lines are almost the same for a set of parameters, we can call the average gap the period $T$. For example, the periods $T$ for the three cases shown in Fig. 1 are 3.13, 3.22 and 3.42, respectively. The period for a special mass spectrum increases with $\lambda$. Figure 2 states the effect of the size of the extra dimen-

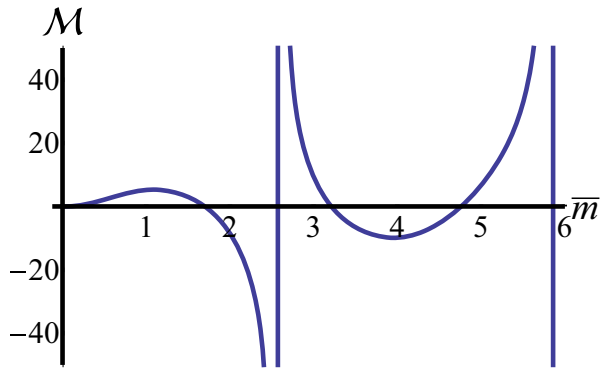

(a)

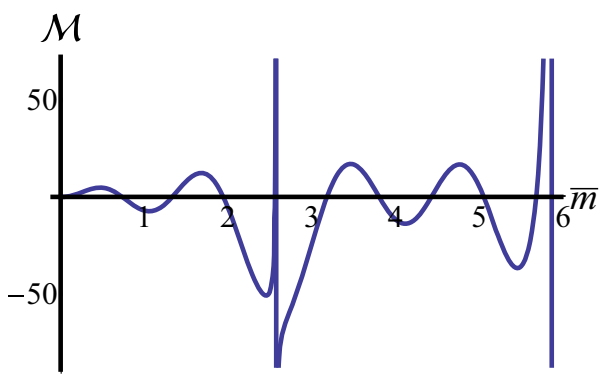

(b)

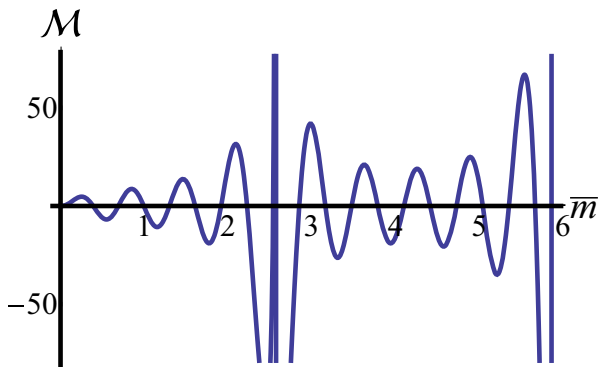

(c)

Fig. 2 The effect of the size of the extra dimension, $\bar{z}_{b}$ (large $\bar{z}_{b}>1$ ), on the mass spectra of the scalar KK modes. The other parameters are set to $k=-3$ and $\lambda=5$

sion, $\bar{z}_{b}$, on the gap of the massive KK modes. It shows that the number of excited states in a single period increases with $\bar{z}_{b}$, and the gap of massive KK modes decreases with $\bar{z}_{b}$. An interesting phenomenon will appear if we set $\bar{z}_{b}<1$ (and $k=-3$ and $\lambda=5$ at the same time). The massive KK modes will not appear in every period anymore for this case; however, they will appear after severe periods, as shown in Fig. 3. The mass spectra of the scalar KK modes are shown in Fig. 4 for different parameters. The mass gap between the zero mode and the first massive KK mode increases with the dilaton coupling constant $\lambda$ and decreases with the size of the extra dimension $\bar{z}_{b}$. On the other hand, the mass spectrum is almost equidistant for the higher excited states, and relatively sparse for the lower excited states.

\subsubsection{The Higgs potential coupling}

Next, we consider another coupling, the Higgs potential type coupling between a real scalar $\Phi$ and the background 


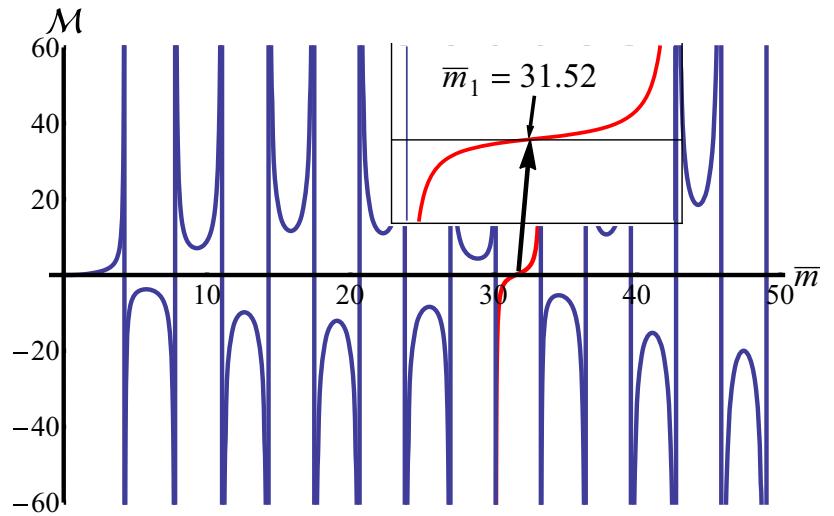

(a)

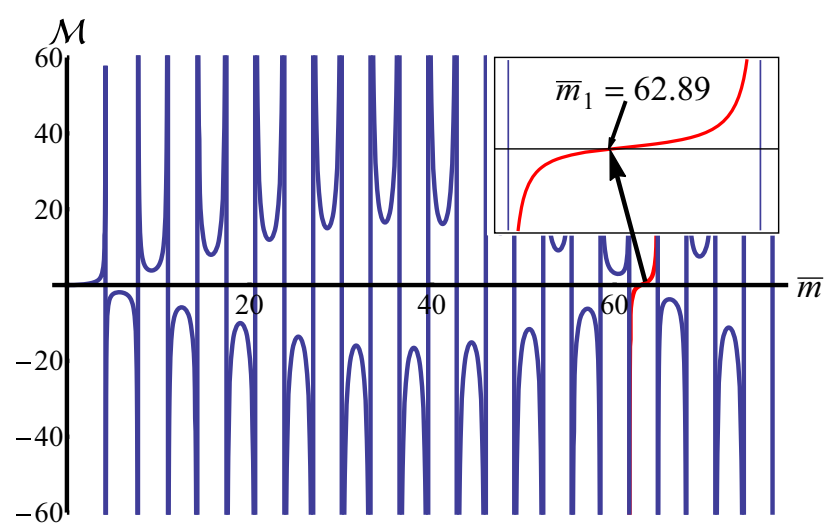

(b)

Fig. 3 The effect of the size of the extra dimension, $\bar{z}_{b}\left(\operatorname{small} \bar{z}_{b}<1\right.$ ), on the mass spectra of the scalar KK modes. The other parameters are set to $k=-3, \lambda=5$

scalar field $\omega$. The five-dimensional action is assumed as [14],

$S_{\mathrm{s}}=\int \mathrm{d}^{5} x \sqrt{-g}\left[-\frac{1}{2} g^{M N} \partial_{M} \Phi \partial_{N} \Phi-V(\Phi, \omega)\right]$,

where the Higgs potential $V(\Phi, \omega)$ is given by

$V(\Phi, \omega)=\left(\theta \omega^{2}-u^{2}\right) \Phi^{2}$

According to the metric (14) and the action (34), the equation of motion of the five-dimensional real scalar $\Phi$ reads

$\partial_{\mu} \partial^{\mu} \Phi+e^{-3 A} \partial_{z}\left(e^{3 A} \partial_{z} \Phi\right)-2 e^{2 A}\left(\theta \omega^{2}-u^{2}\right) \Phi=0$.

Then, making the KK decomposition $\Phi\left(x^{\mu}, z\right)=$ $\sum_{n} \phi_{n}\left(x^{\mu}\right) \chi_{n}(z) e^{-3 A / 2}$ and demanding that $\phi_{n}\left(x^{\mu}\right)$ satisfies the four-dimensional massive Klein-Gordon equation $\left(\partial_{\mu} \partial^{\mu}-m_{n}^{2}\right) \phi_{n}=0$, we obtain the Schrödinger-like equation of the extra-dimensional part $\chi_{n}(z)$,

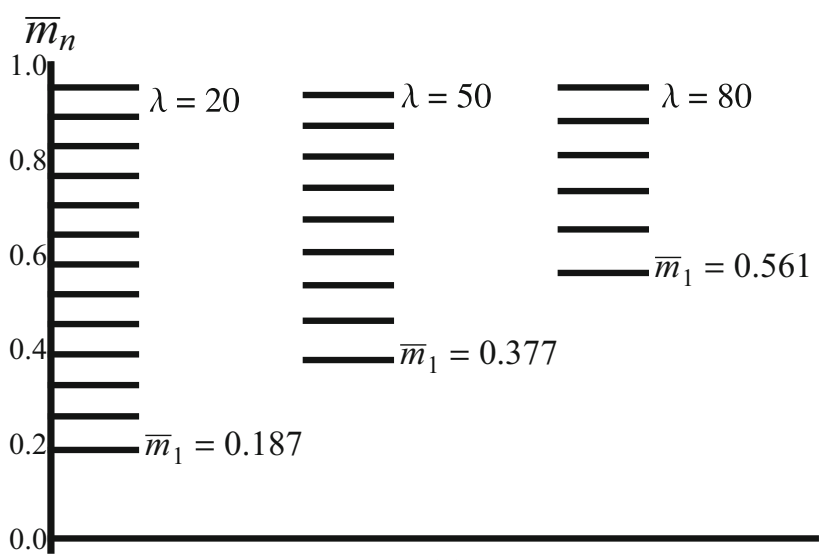

(a)

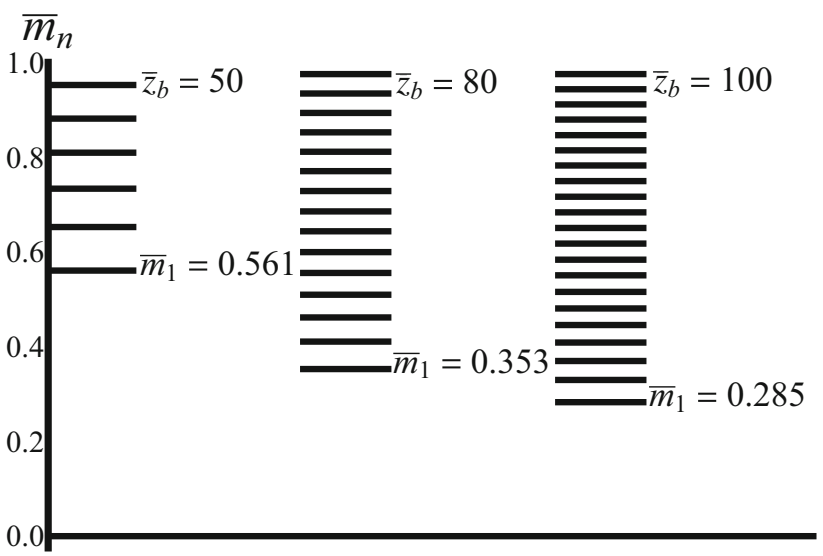

(b)

Fig. 4 The mass spectra of the scalar KK modes. The parameter $k$ is set to $k=-3$

$\left[-\partial_{z}^{2}+V_{\mathrm{s}}(z)\right] \chi_{n}(z)=m_{n}^{2} \chi_{n}(z)$

Here $m_{n}$ is the mass of the $n$th KK mode, and the effective potential $V_{\mathrm{s}}(z)$ is given by

$V_{\mathrm{s}}(z)=\frac{3}{2} \partial_{z}^{2} A+\frac{9}{4}\left(\partial_{z} A\right)^{2}+2 e^{2 A}\left(\theta \omega^{2}-u^{2}\right)$.

The fundamental five-dimensional action (34) can be reduced to the effective four-dimensional action of the massive scalars, when $\chi_{n}$ satisfy the following orthonormality conditions:

$\int_{-z_{b}}^{+z_{b}} \chi_{m}(z) \chi_{n}(z) \mathrm{d} z=\delta_{m n}$

The explicit expression of the effective potential $V_{\mathrm{s}}(z)$ for the brane solution (10) is 


$$
\begin{aligned}
V_{\mathrm{S}}(z)= & \frac{\left(k^{2}-\alpha^{2}\right) \beta^{2}}{4 \alpha^{2}(1+\beta|z|)^{2}} \\
& +2(1+\beta|z|)^{\frac{2(\alpha+k)}{3 \alpha}}\left(\frac{\theta}{\alpha^{2}} \ln (1+\beta|z|)-u^{2}\right) \\
& +\frac{k+\alpha}{\alpha} \beta\left[\delta(z)-\frac{1}{1+\beta z_{b}} \delta\left(z-z_{b}\right)\right] .
\end{aligned}
$$

At $z=0$, the value of $V_{\mathrm{s}}(z)$ is

$V_{\mathrm{S}}(0)=\frac{\left(k^{2}-\alpha^{2}\right) \beta^{2}-8 u^{2} \alpha^{2}}{4 \alpha^{2}}+\frac{k+\alpha}{\alpha} \beta \delta(0)$.

Considering that $\beta>0, k<-1$ and $\sqrt{3} / 2<\alpha=$ $\sqrt{k^{2}+3 k+3}<-k$, we know that the effective potential $V_{\mathrm{s}}(z)$ is negative at $z=0$. Therefore, the scalar zero mode can be localized on the positive tension brane.

For convenience, we redefine some new dimensionless parameters:

$|\bar{z}| \equiv \beta|z|, \quad \bar{\theta} \equiv \frac{\theta}{\beta^{2}}, \quad \bar{u} \equiv \frac{u}{\beta}, \quad \bar{m}_{n} \equiv \frac{m_{n}}{\beta}$,

and the Schrödinger-like equation (37) is changed as

$\left(-\partial_{\bar{z}}^{2}+\bar{V}_{\mathrm{s}}\right) \chi(\bar{z})=\bar{m}_{n}^{2} \chi(\bar{z})$

where the expression of the dimensionless effective potential $\bar{V}_{\mathrm{s}}$ is

$$
\begin{aligned}
\bar{V}_{\mathrm{s}}(\bar{z}) \equiv & \frac{V_{\mathrm{s}}}{\beta^{2}}=\frac{\left(k^{2}-\alpha^{2}\right)}{4 \alpha^{2}(1+|\bar{z}|)^{2}} \\
& +2(1+|\bar{z}|)^{\frac{2(\alpha+k)}{3 \alpha}}\left(\frac{\bar{\theta}}{\alpha^{2}} \ln (1+|\bar{z}|)-\bar{u}^{2}\right) \\
& +\frac{k+\alpha}{\alpha}\left[\delta(\bar{z})-\frac{1}{1+\bar{z}_{b}} \delta\left(\bar{z}-\bar{z}_{b}\right)\right] .
\end{aligned}
$$

At the boundary $\bar{z}=\bar{z}_{b}$, the effective potential $\bar{V}_{\mathrm{s}}(\bar{z})(44)$ has a positive $\delta$ function (just as an infinite high barrier), and it will yield infinite discrete bound KK modes. Due to the nonanalytical solution of the Schrödinger-like equation (43), we can only solve numerically Eq. (43) by fixing the parameter $\bar{\theta}$ and choosing the proper value $\bar{u}$, to ensure the zero mode can be localized on the brane.

The wave functions of the lower bound KK modes are plotted in Fig. 5 and the corresponding mass spectrum is listed as follows:

$$
\begin{aligned}
\bar{m}_{n}= & (0,2.84,4.50,6.09,7.68, \\
& 9.25,10.81,12.36,13.91,15.46, \ldots),
\end{aligned}
$$

where the parameters are set to $k=-2, \bar{\theta}=4.77, \bar{z}_{b}=1$, and $\bar{u}=1.43$. From the numerical solution above, we know that the zero mode can be trapped on the brane by fine tuning the parameters. We can see that the mass spectrum would get denser for the higher excited states, while they get sparser for the lower excited states, and the gap of the higher excited states is almost the same for a set of parameters, on account of the dimensionless effective potential $\bar{V}_{\mathrm{s}}(\bar{z})$ at the boundary

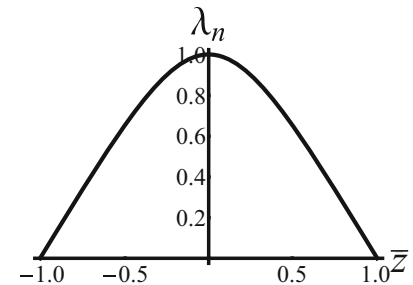

(a)

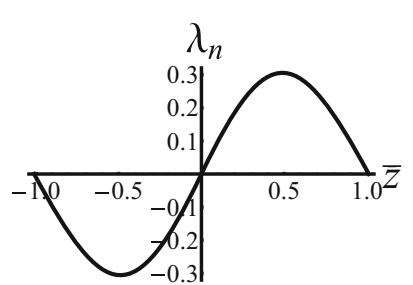

(b)

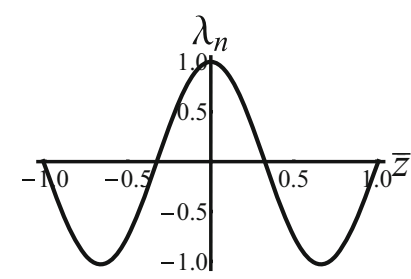

(c)

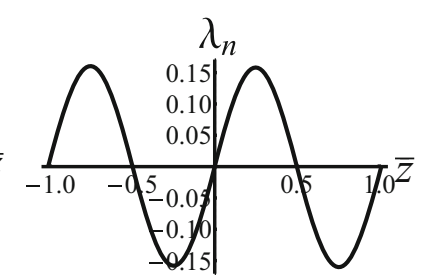

(d)
Fig. 5 The wave functions of the lower scalar KK modes. The parameters are set to $k=-2, \bar{\theta}=4.77, \bar{z}_{b}=1$, and $\bar{u}=1.43$

$\bar{z}= \pm \bar{z}_{b}$ having two positive $\delta$ functions, and the effective potential $\bar{V}_{\mathrm{s}}(\bar{z})$ just like an infinite high barrier. Therefore, the solutions at the high excited states should be trigonometric functions with the same mass gap. By increasing the value of the parameter $\bar{u}$, the bound state solution with $\bar{m}_{n}^{2}<0$ will appear. Therefore, in order to obtain nonnegative eigenvalues $\bar{m}^{2}$, the mass parameter $\bar{u}$ will have an upper limit when the other parameters are fixed. For $k=-2, \bar{\theta}=4.77, \bar{z}_{b}=1$, as we have chosen here, the upper limit of $\bar{u}$ is 1.43 .

\subsection{Spin-1 vector field}

Secondly, we turn to the spin-1 vector field. A typical fivedimensional action of a vector field coupled with a dilaton field $\omega$ is given as

$S_{\mathrm{V}}=-\frac{1}{4} \int \mathrm{d}^{5} x \sqrt{-g} e^{\tau \omega} g^{M N} g^{R S} F_{M R} F_{N S}$,

where $F_{M N}=\partial_{M} A_{N}-\partial_{N} A_{M}$ is the field strength as usual and $\tau$ is a dimensionless coupling constant. Considering the explicit form of the metric (14), the equations of motion read

$$
\begin{aligned}
e^{\tau \omega} \partial_{\nu} F^{\mu \nu}+\eta^{\mu \lambda} a^{-1}(z) \partial_{4}\left(a(z) e^{\tau \omega} F_{4 \lambda}\right) & =0, \\
e^{\tau \omega} \partial_{\mu}\left(\eta^{\mu \nu} F_{\nu 4}\right) & =0 .
\end{aligned}
$$

In order to be consistent with the gauge invariant equation $\oint \mathrm{d} z A_{4}=0$, we use the gauge freedom to choose $A_{4}=0$. Under the $\mathrm{KK}$ decomposition of the vector field

$A_{\mu}\left(x^{\mu}, z\right)=\sum_{n} a_{\mu}^{(n)}\left(x^{\mu}\right) \rho_{n}(z) e^{-\epsilon A(z)}$,

where $\epsilon=\frac{k+\alpha-3 \tau}{2(k+\alpha)}$, and the orthonormality conditions

$\int_{-z_{b}}^{+z_{b}} \rho_{m}(z) \rho_{n}(z) \mathrm{d} z=\delta_{m n}$ 
the action (46) can be reduced to an effective one including the four-dimensional massless vector field (the zero mode) and a set of massive vector fields (the massive KK modes):

$S_{\mathrm{v}}=\sum_{n} \int \mathrm{d}^{4} x\left(-\frac{1}{4} f_{\mu \nu}^{(n)} f^{\mu \nu(n)}-\frac{1}{2} m_{n}^{2} a_{\mu}^{(n)} a^{\mu(n)}\right)$,

where $f_{\mu \nu}^{(n)}=\partial_{\mu} a_{\nu}^{(n)}-\partial_{\nu} a_{\mu}^{(n)}$ is the field strength of the fourdimensional vector field. In addition, the extra-dimensional part $\rho_{n}(z)$ satisfies the following Schrödinger-like equation:

$\left[-\partial_{z}^{2}+V_{\mathrm{v}}(z)\right] \rho_{n}(z)=m_{n}^{2} \rho_{n}(z)$,

where $m_{n}$ is the mass of the vector KK mode and the effective potential $V_{\mathrm{v}}(z)$ is

$V_{\mathrm{v}}(z)=\left(\epsilon \partial_{z} A\right)^{2}+\epsilon \partial_{z}^{2} A$.

The explicit expression of the effective potential $V_{\mathrm{v}}(z)$ reads

$$
\begin{aligned}
V_{\mathrm{v}}(z)= & \frac{b(k+\alpha-3 \tau) \beta^{2}}{36 \alpha^{2}(1+\beta|z|)^{2}} \\
& +\frac{(k+\alpha-3 \tau) \beta}{3 \alpha}\left[\delta(z)-\frac{\delta\left(z-z_{b}\right)}{1+\beta z_{b}}\right],
\end{aligned}
$$

where $b=(k-5 \alpha-3 \tau)$. The value of the effective potential $V_{\mathrm{v}}(z)$ at $z=0$ is

$V_{\mathrm{v}}(0)=\frac{b(k+\alpha-3 \tau) \beta^{2}}{36(k+\alpha) \alpha^{2}}+\frac{(k+\alpha-3 \tau) \beta}{3 \alpha} \delta(0)$.

For localizing the vector zero mode on the positive tension brane, the effective potential $V_{\mathrm{v}}(z)$ should be negative at $z=$ 0 , which is equivalent to the following condition:

$\tau>\frac{1}{3}(k+\alpha)$.

By setting $m=0$ in Eq. (51), we can get the normalized zero mode of the vector field for $\tau \neq \frac{k+4 \alpha}{3}$ :

$\rho_{0}(z)=\sqrt{\frac{\beta d}{6 \alpha\left[\left(1+\beta z_{b}\right)^{\frac{d}{3 \alpha}}-1\right]}}(1+\beta|z|)^{\frac{k+\alpha-3 \tau}{6 \alpha}}$,

where $d=k+4 \alpha-3 \tau$. It is localized on the positive tension brane when the extra dimension is finite under the condition (55). It is easy to see that the vector zero mode can also be localized on the brane when $\tau=0$ if the extra dimension is finite. If the parameter $\tau=\frac{k+4 \alpha}{3}$, the normalized vector zero mode is

$\rho_{0}(z)=\sqrt{\frac{\beta}{2 \ln \left(1+\beta z_{b}\right)}} \frac{1}{\sqrt{1+\beta|z|}} \quad\left(\tau=\frac{k+4 \alpha}{3}\right)$.

The vector zero mode (57) can be localized on the positive tension brane only for the case of a finite extra dimension. However, we note here that if the extra dimension is infinite, the zero mode can also be localized on this brane for $\tau>$ $\frac{k+4 \alpha}{3}$.
By defining the dimensionless potential

$\bar{V}_{v}(\bar{z})=\frac{V_{v}(z)}{\beta^{2}}$,

Eq. (51) can be rewritten in a dimensionless form,

$\left[-\partial_{\bar{z}}^{2}+\bar{V}_{v}(\bar{z})\right] \rho_{n}(\bar{z})=\bar{m}_{n}^{2} \rho_{n}(\bar{z})$,

where the dimensionless parameters $\bar{z}$ and $\bar{m}_{n}$ are defined as in Eq. (26). For simplicity, we only require that the KK modes satisfy the Neumann boundary condition $\partial_{\bar{z}}\left(e^{-\epsilon A(\bar{z})} \rho_{n}\right)=0$ at the boundaries $\bar{z}=0$ and $\bar{z}=\bar{z}_{b}$. Using the boundary condition at $\bar{z}=0$, we get the general solution of Eq. (51),

$\rho_{n}(\bar{z})=N(1+|\bar{z}|)^{\frac{1}{2}}\left[\mathrm{~J}_{P_{\mathrm{v}}}\left(\bar{m}_{n}(|\bar{z}|+1)\right)+\mathcal{C}_{\mathrm{v}} \mathrm{Y}_{P_{\mathrm{v}}}\left(\bar{m}_{n}(|\bar{z}|+1)\right)\right]$,

where $N$ is the normalization coefficient and

$\mathcal{C}_{\mathrm{v}} \equiv-\frac{\mathrm{J}_{P_{\mathrm{v}}-1}\left(\bar{m}_{n}\right)}{\mathrm{Y}_{P_{\mathrm{v}}-1}\left(\bar{m}_{n}\right)}, \quad P_{\mathrm{v}} \equiv \frac{2 \alpha+3 \tau-k}{6 \alpha}$.

With another boundary condition at $\bar{z}=\bar{z}_{b}$, we obtain the spectrum of the vector KK modes determined by the following condition:

$$
\begin{aligned}
\mathcal{M}(\bar{m}) \equiv & \mathbf{J}_{P_{\mathrm{v}}-1}\left(\bar{m}_{n}\left(\bar{z}_{b}+1\right)\right) \\
& +\mathcal{C}_{\mathrm{v}} \mathrm{Y}_{P_{\mathrm{v}}-1}\left(\bar{m}_{n}\left(\bar{z}_{b}+1\right)\right)=0 .
\end{aligned}
$$

We plot the relation between $\mathcal{M}$ and $\bar{m}$ In Figs. 6 and 7 for large and small $\bar{z}_{b}$, respectively. Figure $6 \mathrm{a}, \mathrm{b}$ imply that the mass of the first excited state increases with the coupling constant of dialton, $\tau$. Figure $6 \mathrm{~b}, \mathrm{c}$ show that in a single cycle the number for the excited states increases with the size of the extra dimension, $\bar{z}_{b}$. In Fig. 7, we adjust the size of the extra dimension $\bar{z}_{b}=0.1$ and $\bar{z}_{b}=0.06$ and let $k=-3, \tau=20$ at the same time; the result shows that the excited states do not exist in each cycle and they only emerge after multiple periods. In addition, Fig. 8 shows that the spectrum interval approaches a constant for the higher excited states, while for the lower excited states it is relatively sparse.

\subsection{Spin- $1 / 2$ fermion field}

Furthermore, we investigate the localization and mass spectrum of spin- $1 / 2$ fermions on the Weyl brane. For studying the localization of fermions on thick branes, Refs. [11,16,4552,52-55] have introduced the Yukawa coupling. Here, we consider the following five-dimensional Dirac action for a fermion coupled with the background scalar field $\omega$ :

$S_{\frac{1}{2}}=\int \mathrm{d}^{5} x \sqrt{-g}\left[\bar{\Psi} \Gamma^{M}\left(\partial_{M}+\omega_{M}\right) \Psi-\eta \bar{\Psi} F(\omega) \Psi\right]$, 


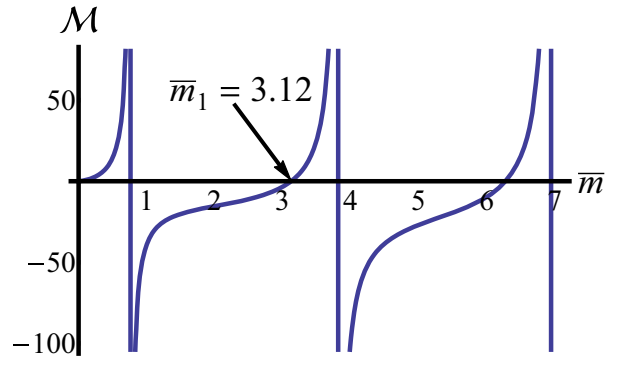

(a)

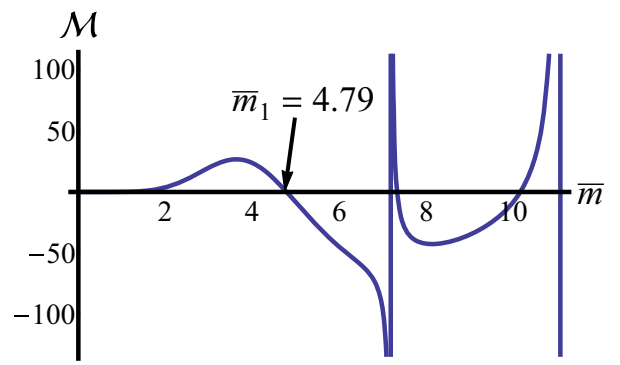

(b)

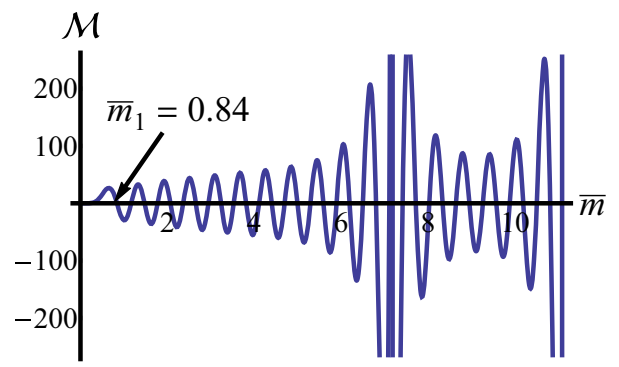

(c)

Fig. 6 The effect of the parameter $\tau$ and the size of the extra dimension $\bar{z}_{b}$ on the massive vector KK modes, and the parameter $k$ is set to $k=-3$

where $\omega_{M}$ is the spin connection defined as $\omega_{M}=\frac{1}{4} \omega_{M}^{\bar{M} \bar{N}} \Gamma_{\bar{M}} \Gamma_{\bar{N}}$ with $\omega_{M}^{\bar{M} \bar{N}}$ given by

$$
\begin{aligned}
\omega_{M}^{\bar{M}} \bar{N}= & \frac{1}{2} e^{N \bar{M}}\left(\partial_{M} e_{N}^{\bar{N}}-\partial_{N} e_{M}^{\bar{N}}\right) \\
& -\frac{1}{2} e^{N \bar{N}}\left(\partial_{M} e_{N}^{\bar{M}}-\partial_{N} e_{M}^{\bar{M}}\right) \\
& -\frac{1}{2} e^{P \bar{M}} e^{Q \bar{N}}\left(\partial_{P} e_{Q \bar{R}}-\partial_{Q} e_{P \bar{R}}\right) e_{M}^{\bar{R}} .
\end{aligned}
$$

The non-vanishing components of the spin connection $\omega_{M}$ for the background metric (14) are

$\omega_{\mu}=\frac{1}{2}\left(\partial_{z} A\right) \gamma_{\mu} \gamma_{5}+\hat{\omega}_{\mu}$,

where $\hat{\omega}_{\mu}=\frac{1}{4} \bar{\omega}_{\mu}^{\bar{\mu} \bar{\nu}} \Gamma_{\bar{\mu}} \Gamma_{\bar{\nu}}$ is the spin connection derived from the metric $\hat{g}_{\mu \nu}(x)=\hat{e}_{\mu}^{\bar{\mu}}(x) \hat{e}_{v}{ }^{\bar{v}}(x) \eta_{\bar{\mu}} \bar{v}$. Then we can obtain the equation of motion

$$
\left\{\gamma^{\mu}\left(\partial_{\mu}+\hat{\omega}_{\mu}\right)+\gamma^{5}\left(\partial_{z}+2 \partial_{z} A\right)-\eta \mathrm{e}^{A} F(\omega)\right\} \Psi=0,
$$

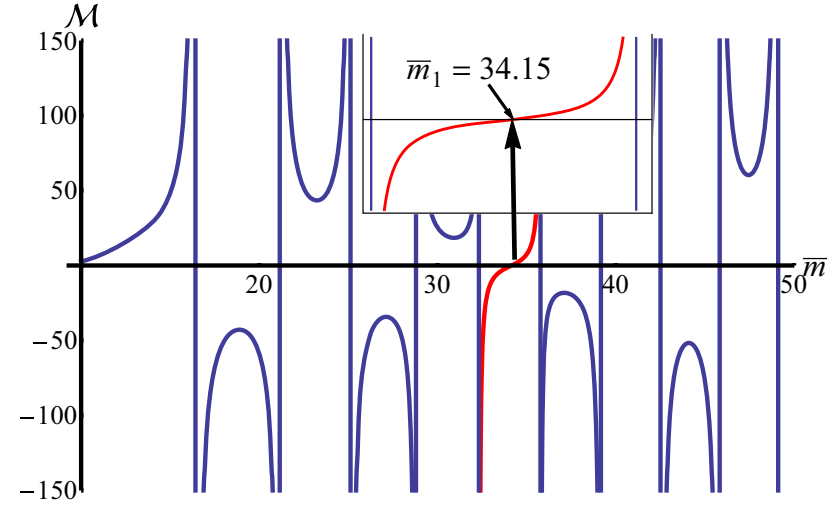

(a)

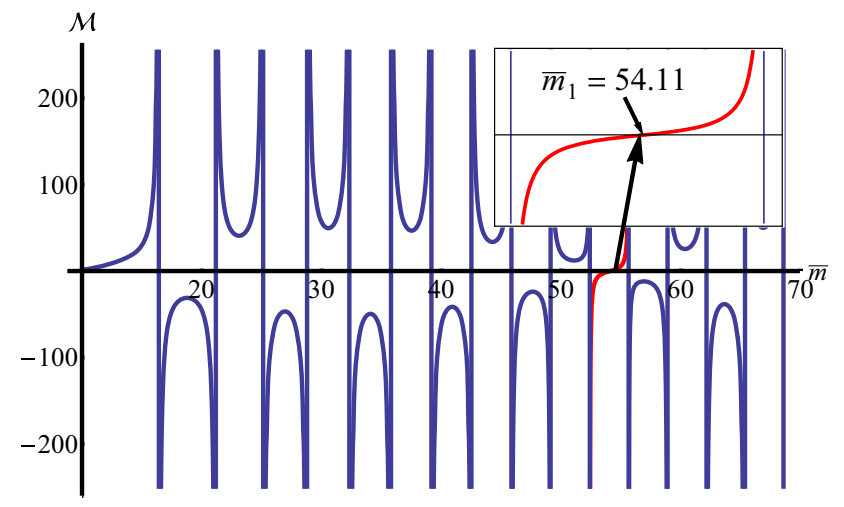

(b)

Fig. 7 The effect of the size of the extra dimension $\bar{z}_{b}\left(\right.$ small $\left.\bar{z}_{b}<1\right)$ on the massive vector KK modes. The parameters are set to $k=-3$, and $\tau=20$

where $\gamma^{\mu}\left(\partial_{\mu}+\hat{\omega}_{\mu}\right)$ is the Dirac operator on the brane. For the current case of a flat brane, the spin connection on the brane vanishes, i.e., $\hat{\omega}_{\mu}=0$.

We make a general chiral decomposition,

$\Psi=\mathrm{e}^{-2 A(z)} \sum_{n}\left(\psi_{L n}(x) f_{L n}(z)+\psi_{R n}(x) f_{R n}(z)\right)$,

where $\psi_{L n}(x)=-\gamma^{5} \psi_{L n}(x)$ and $\psi_{R n}(x)=\gamma^{5} \psi_{R n}(x)$ are the left- and right-chiral components of a four-dimensional Dirac field, respectively. Then we can show that $\psi_{L}(x)$ and $\psi_{R}(x)$ satisfy the four-dimensional massive Dirac equations $\gamma^{\mu}\left(\partial_{\mu}+\hat{\omega}_{\mu}\right) \psi_{L n}(x)=m_{n} \psi_{R_{n}}(x)$ and $\gamma^{\mu}\left(\partial_{\mu}+\right.$ $\left.\hat{\omega}_{\mu}\right) \psi_{R n}(x)=m_{n} \psi_{L_{n}}(x)$, and the KK modes $f_{L n}(z)$ and $f_{R n}(z)$ satisfy the following coupled equations:

$\left[\partial_{z}+\eta \mathrm{e}^{A} F(\omega)\right] f_{L n}(z)=m_{n} f_{R n}(z)$,
$\left[\partial_{z}-\eta \mathrm{e}^{A} F(\omega)\right] f_{R n}(z)=-m_{n} f_{L n}(z)$.

From the above coupled equations, we can obtain the Schrödinger-like equations for the left- and right-chiral KK modes of the fermion 


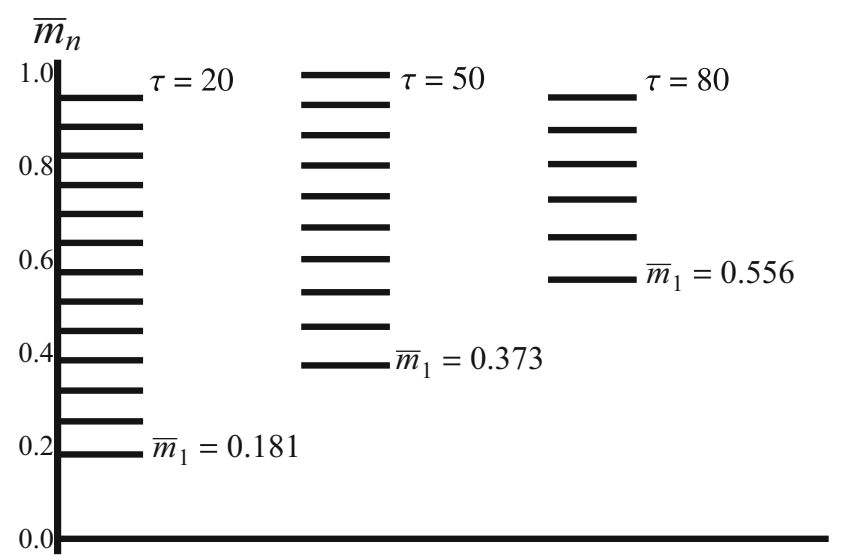

(a)

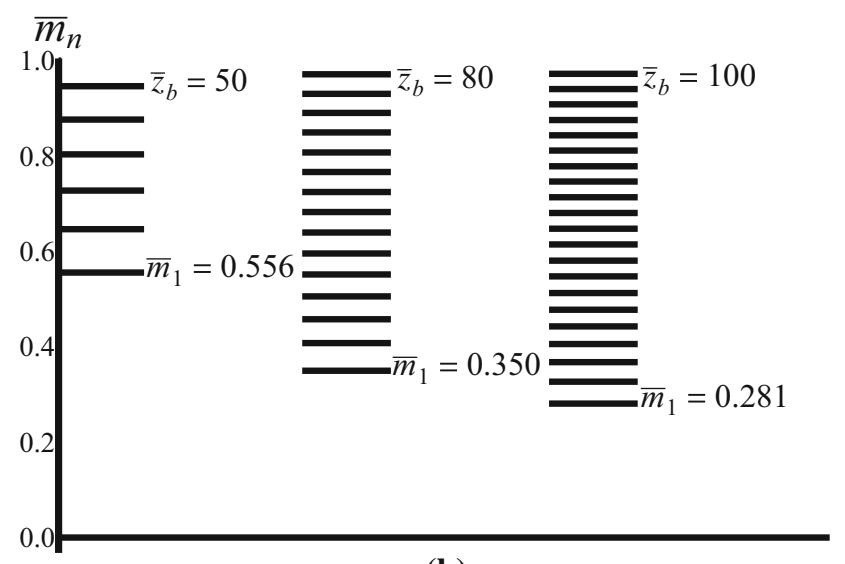

(b)

Fig. 8 The mass spectra of the vector KK modes. The parameter $k$ is set to $k=-3$

$\left(-\partial_{z}^{2}+V_{L}(z)\right) f_{L n}=m_{L_{n}}^{2} f_{L n}$,

$\left(-\partial_{z}^{2}+V_{R}(z)\right) f_{R n}=m_{R_{n}}^{2} f_{R n}$,

where the effective potentials are given by

$$
\begin{aligned}
V_{L}(z) & =\left(\eta \mathrm{e}^{A} F(\omega)\right)^{2}-\partial_{z}\left(\eta \mathrm{e}^{A} F(\omega)\right), \\
V_{R}(z) & =\left.V_{L}(z)\right|_{\eta \rightarrow-\eta} .
\end{aligned}
$$

In order to get the effective four-dimensional action for a massless fermion and a set of massive Dirac fermions

$$
S_{\frac{1}{2}}=\sum_{n} \int \mathrm{d}^{4} x \sqrt{-\hat{g}} \bar{\psi}_{n}\left[\gamma^{\mu}\left(\partial_{\mu}+\hat{\omega}_{\mu}\right)-m_{n}\right] \psi_{n}
$$

we need to introduce the following orthonormality conditions:

$$
\begin{aligned}
& \int_{-z_{b}}^{z_{b}} f_{L m} f_{L n} \mathrm{~d} z=\int_{-z_{b}}^{z_{b}} f_{R m} f_{R n} \mathrm{~d} z=\delta_{m n}, \\
& \int_{-z_{b}}^{z_{b}} f_{L m} f_{R n} \mathrm{~d} z=0 .
\end{aligned}
$$

We set $F(\omega)=\partial_{z}\left(e^{v \omega}\right)$ as a simple example. The explicit expressions of the effective potentials read

$$
\begin{aligned}
V_{L}(z)= & \frac{v \eta \beta^{2}}{\alpha^{2}}(1+\beta|z|)^{\frac{k-5 \alpha-3 v}{3 \alpha}} \\
& \times\left[v \eta(1+\beta|z|)^{\frac{k+\alpha-3 v}{3 \alpha}}+\frac{k-2 \alpha-3 v}{3}\right] \\
& +\frac{2 v \eta \beta}{\alpha}\left[\delta(z)-\frac{1}{1+\beta z_{b}} \delta\left(z-z_{b}\right)\right], \\
V_{R}(z)= & \left.V_{L}(z)\right|_{\eta \rightarrow-\eta} .
\end{aligned}
$$

One can easily calculate the values of $V_{L}(z)$ and $V_{R}(z)$ at $z=0$ :

$V_{L}(0)=\frac{v \eta \beta^{2}}{\alpha^{2}}\left[v \eta-v-\alpha+\frac{1}{3}(k+\alpha)\right]+\frac{2 v \eta \beta}{\alpha} \delta(0)$,

$V_{R}(0)=\left.V_{L}(0)\right|_{\eta \rightarrow-\eta}$.

It can be shown that the left- and right-chiral fermion zero modes cannot be localized on the positive tension brane at the same time. For localizing the zero mode of the left-chiral fermion on the positive tension brane, the effective potential $V_{L}(z)$ should be negative at $z=0$, which requires that $\eta v<$ 0 , since $\beta$ and $\alpha$ are positive.

In the following, we suppose the left-chiral fermion has the zero mode localized on the positive tension brane. So we only consider the left-chiral fermion (the right-chiral fermion will have the same mass spectrum with $m_{n}>0$ ). The solution of the left-chiral fermion zero mode is

$$
f_{L 0}(z) \propto \exp \left(-\eta \int \mathrm{d} z^{\prime} e^{A\left(z^{\prime}\right)} F(\omega)\right) .
$$

For the case of an infinite extra dimension, in order to localize the zero mode $f_{L 0}(z)$ on the brane, the following normalization condition should be satisfied:

$\int_{-\infty}^{\infty} \mathrm{d} z f_{L 0}^{2}(z) \propto \int_{-\infty}^{\infty} e^{\left(-2 \eta \int^{z} \mathrm{~d} \bar{z} e^{A(\bar{z})} F(\bar{\omega})\right)} \mathrm{d} z<\infty$

which turns out to be $v<\frac{1}{3}(k+\alpha)(<0)$ and $\eta>0$. Next, we consider the case of finite extra dimension, for which we only need $\eta v<0$ in order for the zero mode $f_{L 0}$ to be localized on the positive tension brane. We will investigate the localization and mass spectrum for the special case $v=\frac{1}{3}(k+\alpha)<0$. The effective potential for the left-chiral fermion KK modes is reduced to

$$
\begin{aligned}
V_{L}(z)= & \frac{v \eta \beta^{2}}{\alpha^{2}(1+\beta|z|)^{2}}(v \eta-\alpha) \\
& +\frac{2 v \eta \beta}{\alpha}\left[\delta(z)-\frac{1}{1+\beta z_{b}} \delta\left(z-z_{b}\right)\right] .
\end{aligned}
$$

Now, in order to transform Eq. (68) into a dimensionless one, we redefine the following dimensionless potential:

$\bar{V}_{L}(\bar{z})=\frac{V_{L}(z)}{\beta^{2}}$. 
We assume the KK modes satisfy the Neumann boundary condition $\partial_{\bar{z}}\left(e^{-2 A(\bar{z})} f_{L n}\right)=0$ at the boundaries $\bar{z}=0$ and $\bar{z}=\bar{z}_{b}$. By using the boundary condition at $\bar{z}=0$, we get the general solution of Eq. (68),

$f_{L n}(\bar{z})=N(1+|\bar{z}|)^{\frac{1}{2}}\left[\mathrm{~J}_{P_{\mathrm{f}}}(\bar{z})+\mathcal{C}_{\mathrm{f}} \mathrm{Y}_{P_{\mathrm{f}}}(\bar{z})\right]$,

where

$\mathcal{C}_{\mathrm{f}} \equiv-\frac{\mathrm{J}_{P_{\mathrm{f}}-1}\left(\bar{m}_{n}\right)}{\mathrm{Y}_{P_{\mathrm{f}}-1}\left(\bar{m}_{n}\right)}, \quad P_{\mathrm{f}} \equiv \frac{\sqrt{(-3 \alpha+2 k \eta+2 \alpha \eta)^{2}}}{6 \alpha}$.

Another boundary condition will give the mass spectrum of the fermion KK modes:

$\mathcal{M} \equiv\left(4 k+\alpha+6 P_{\mathrm{f}} \alpha\right) B_{P_{\mathrm{f}}}-\left(4 \bar{m}_{n} \alpha \bar{z}_{b}\right) B_{P_{\mathrm{f}-1}}=0$,

where $B_{P_{\mathrm{f}}}$ and $B_{P_{\mathrm{f}-1}}$ are defined as follows:

$$
\begin{aligned}
& B_{P_{\mathrm{f}}} \equiv \mathrm{J}_{P_{\mathrm{f}}}\left(\bar{m}_{n}\left(\bar{z}_{b}+1\right)\right)+\mathcal{C}_{\mathrm{f}} \mathrm{Y}_{P_{\mathrm{f}}}\left(\bar{m}_{n}\left(\bar{z}_{b}+1\right)\right), \\
& B_{P_{\mathrm{f}-1}} \equiv \mathrm{J}_{P_{\mathrm{f}}-1}\left(\bar{m}_{n}\left(\bar{z}_{b}+1\right)\right)+\mathcal{C}_{\mathrm{f}} \mathrm{Y}_{P_{\mathrm{f}}-1}\left(\bar{m}_{n}\left(\bar{z}_{b}+1\right)\right) .
\end{aligned}
$$

We can obtain the mass spectrum of the left-chiral fermions by numerical calculation, and show some results in Figs. 9, 10, and 11. Figure 9 shows that the mass of the first massive mode increases with the value of the Yukawa coupling constant $\eta$, and the number of the excited states in a single period increases with the size of the extra dimension $\bar{z}_{b}$. Figure 10 indicates that, for the case of $\bar{z}_{b}<1$, the excited states will not appear in every period and they only emerge after multiple periods. We plot the mass spectrum in Fig. 11 for different parameters. It can be seen that the spectrum interval approaches a constant for the higher excited states due to the plane wave behavior of the wave function, while it is relatively sparse for the lower excited states.

\section{Correction to Newtonian potential}

Finally, we consider the tensor fluctuations in this model. Now we add a small perturbation $a^{2}(z) h_{\mu \nu}(x, z)$ to the background metric (8):

$\mathrm{d} s^{2}=a^{2}(z)\left[\left(\eta_{\mu \nu}+h_{\mu \nu}(x, z)\right) \mathrm{d} x^{\mu} \mathrm{d} x^{\nu}+\mathrm{d} z^{2}\right]$.

Following Ref. [67], we suppose the tensor perturbation $h_{\mu \nu}$ satisfies the transverse-traceless (TT) condition: $h_{\mu}^{\mu}=$ $\partial^{\nu} h_{\mu \nu}=0$. The equation of motion for $h_{\mu \nu}$ is given by [61]

$h_{\mu \nu}^{\prime \prime}+3 \frac{a^{\prime}}{a} h_{\mu \nu}^{\prime}+k \omega^{\prime} h_{\mu \nu}^{\prime}+\square^{(4)} h_{\mu \nu}=0$.

Furthermore, $h_{\mu \nu}$ can be decomposed in the form

$h_{\mu \nu}(x, z)=\sum_{n} \varepsilon_{\mu \nu}^{(n)}(x) H^{-\frac{3}{2}}(z) \Psi_{n}(z)$,

where $H(z)=(1+\beta|z|)^{1 / 3}$. The four-dimensional mass $m_{n}$ of a KK excitation is defined by the Klein-Gordon equa-

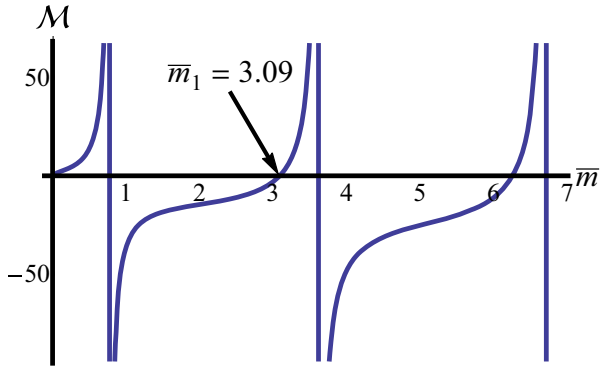

(a)
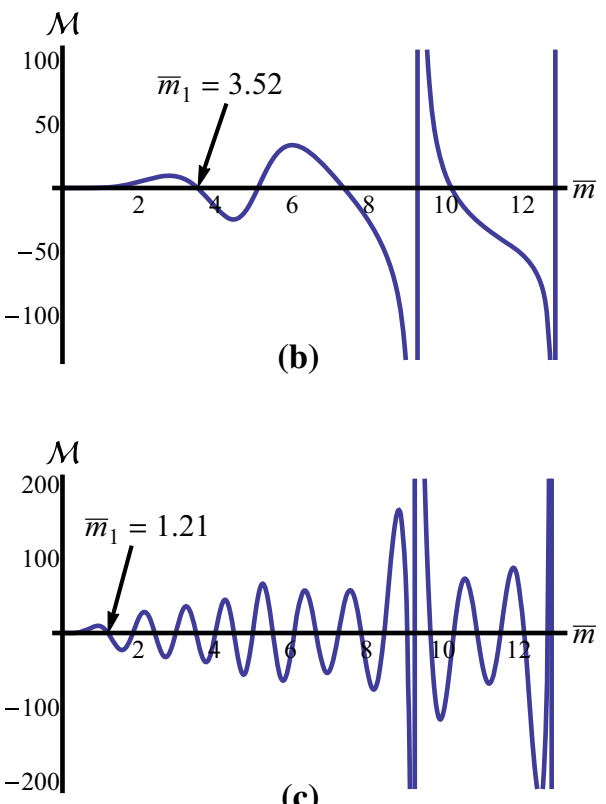

(c)

Fig. 9 The effect of the Yukawa coupling constant $\eta$ and the size of the extra dimension $\bar{z}_{b}$ on the mass spectrum of the left-chiral fermion KK modes. The parameter $k$ is set to $k=-3$

tion $\square^{(4)} \varepsilon_{\mu \nu}^{(n)}(x)=m_{n}^{2} \varepsilon_{\mu \nu}^{(n)}(x)$. We obtain a Schrödinger-like equation from Eq. (87),

$-\Psi_{n}^{\prime \prime}(z)+V(z) \Psi_{n}(z)=m_{n}^{2} \Psi_{n}(z)$,

where the effective potential $V(z)$ is given by

$$
\begin{aligned}
V(z) & =\frac{3}{2} \frac{H^{\prime \prime}}{H}+\frac{3}{4} \frac{H^{\prime 2}}{H^{2}} \\
& =\frac{-\beta^{2}}{4(1+\beta|z|)^{2}}+\beta \delta(z)-\frac{\beta \delta\left(z-z_{b}\right)}{1+\beta z_{b}} .
\end{aligned}
$$

The general solution of Eq. (89) is a linear combination of the Bessel functions,

$$
\begin{aligned}
\Psi_{n}(z)= & N_{n}\left(\frac{1}{\beta}+|z|\right)^{\frac{1}{2}}\left[J_{0}\left(m_{n}\left(|z|+\frac{1}{\beta}\right)\right)\right. \\
& \left.+\alpha_{n} Y_{0}\left(m_{n}\left(|z|+\frac{1}{\beta}\right)\right)\right] .
\end{aligned}
$$

Imposing the Neumann boundary condition $\partial_{z} h_{\mu \nu}(x, z)=0$ at the boundaries $z=0$ and $z=z_{b}$, we get $\alpha_{n}=$ 


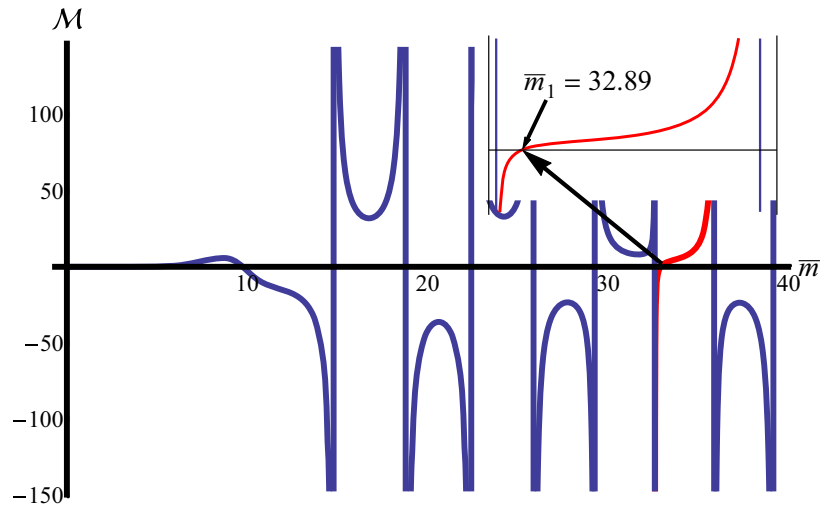

(a)

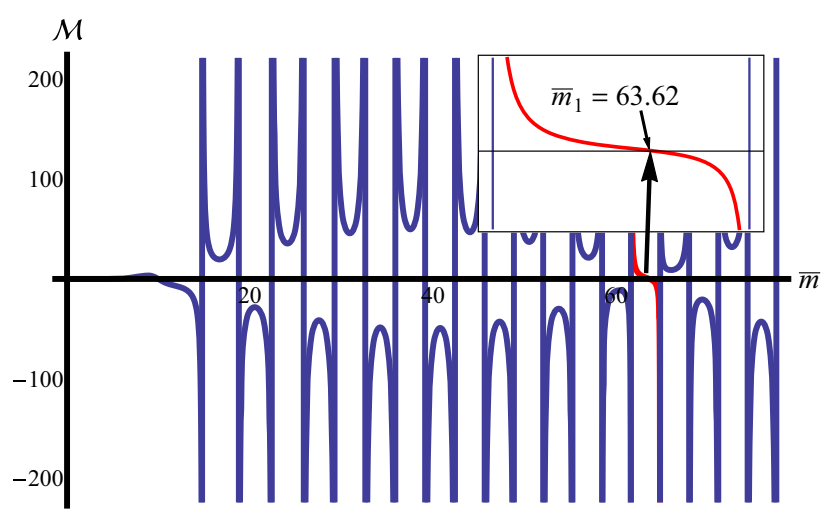

(b)

Fig. 10 The effect of the size of the extra dimension $\bar{z}_{b}\left(\operatorname{small} \bar{z}_{b}<1\right)$ on the mass spectrum of the left-chiral fermion KK modes. The other parameters are set to $k=-3$ and $\eta=40$

$-J_{1}\left(\frac{m_{n}}{\beta}\right) / Y_{1}\left(\frac{m_{n}}{\beta}\right)$ and

$\frac{J_{1}\left(m_{n}\left(z_{b}+\frac{1}{\beta}\right)\right)}{J_{1}\left(\frac{m_{n}}{\beta}\right)}=\frac{Y_{1}\left(m_{n}\left(z_{b}+\frac{1}{\beta}\right)\right)}{Y_{1}\left(\frac{m_{n}}{\beta}\right)}$.

The solution of the graviton zero mode $\Psi_{0}(z)$ is

$\Psi_{0}(z)=N_{0}\left(\frac{1}{\beta}+|z|\right)^{\frac{1}{2}}$

which is localized on the negative tension brane for finite $z_{b}$. Note that the graviton zero mode cannot be normalized anymore when the extra dimension is infinite, which is very different from the RS1 model. The massless zero mode sector of the action (1) leads to the effective four-dimensional gravitational theory,

$$
\begin{aligned}
S_{5} & \supset \frac{M_{*}^{3}}{2} \int_{M_{5}^{W}} \mathrm{~d}^{5} x \sqrt{|g|} e^{k \omega} R \\
& \supset \frac{M_{*}^{3}}{2} \int_{-z_{b}}^{z_{b}} \mathrm{~d} z a^{3}(z) e^{k \omega} \int_{M_{4}} \mathrm{~d}^{4} x \sqrt{\left|g^{(4)}\right|} \hat{R}^{(4)},
\end{aligned}
$$

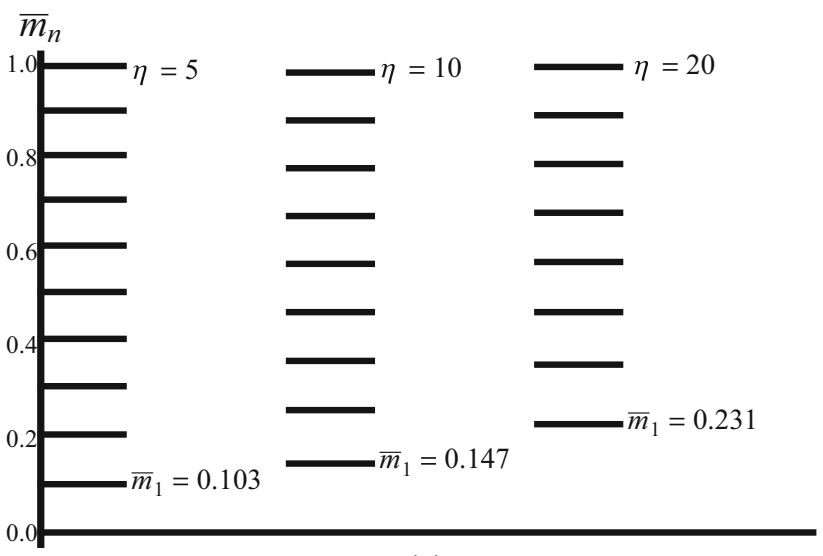

(a)

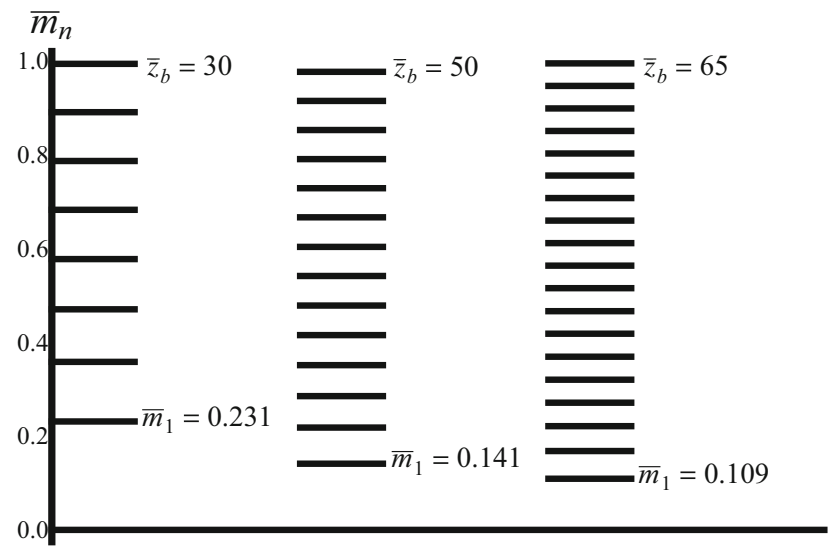

(b)

Fig. 11 The mass spectrum of the left-chiral fermion KK modes for different parameters. The parameter $k$ is set to $k=-3$

where $\hat{R}^{(4)}$ is the four-dimensional Riemannian Ricci scalar made out of $g_{\mu \nu}^{(4)}=\eta_{\mu \nu}+h_{\mu \nu}^{(0)}(x)$. Thus the effective fourdimensional Planck scale $M_{\mathrm{Pl}}$ is [61]

$M_{\mathrm{Pl}}^{2}=M_{*}^{3} \int_{-z_{b}}^{z_{b}} \mathrm{~d} z a^{3}(z) e^{k \omega}=M_{*}^{3}\left(2 z_{b}+\beta z_{b}^{2}\right)$.

For the light modes $\left(m_{n} / \beta \ll 1\right)$, the solution (91) in the long-range limit $\alpha_{n} \approx \frac{\pi m_{n}^{2}}{4 \beta^{2}} \ll 1$ becomes

$\Psi_{n}(z) \approx N_{n}\left(\frac{1}{\beta}+|z|\right)^{\frac{1}{2}}\left[J_{0}\left(m_{n}\left(|z|+\frac{1}{\beta}\right)\right)\right]$

Correspondingly, the spectrum of KK gravitons is [61]

$m_{n}(z)=\frac{x_{n}}{z_{b}+\frac{1}{\beta}} \approx \frac{x_{n}}{z_{b}}$,

where $x_{n}$ satisfies $J_{1}\left(x_{n}\right)=0$, and $x_{1}=3.83, x_{2}=7.02$, $x_{3}=10.17, \ldots$. The normalization constant $N_{n}$ is determined by [68] 
Table 1 Localization conditions for the zero modes of various bulk matter fields. Here $z_{b}$ is the size of the extra dimension

\begin{tabular}{llll}
\hline Bulk matter & Lagrangian & $z_{b}$ & Condition \\
\hline \multirow{2}{*}{ Scalar } & $\mathcal{L}_{0}=-\frac{1}{2} e^{\lambda \omega} \partial_{M} \Phi \partial^{M} \Phi$ & Finite & $\lambda>k+\alpha$ \\
& & Infinite & $\lambda>k+2 \alpha$ \\
Vector & $\mathcal{L}_{1}=-\frac{1}{4} e^{\tau \omega} F_{M N} F^{M N}$ & Finite & $\tau>\frac{1}{3}(k+\alpha)$ \\
& & Infinite & $\tau>\frac{1}{3}(k+4 \alpha)$ \\
Fermion & $\mathcal{L}_{1 / 2}=\bar{\Psi} \Gamma^{M}\left(\partial_{M}+\omega_{M}\right) \Psi-\eta \bar{\Psi} F(\omega) \Psi$ & Finite & $\eta v<0$ \\
& & Infinite & $\eta>0, v<\frac{k+\alpha}{3}$ \\
\hline
\end{tabular}

$1=\int_{-z_{b}}^{z_{b}} \Psi_{n}^{2} \mathrm{~d} z \approx 2 N_{n}^{2} \int_{0}^{z_{b}} z J_{0}\left(m_{n} z\right)^{2} \mathrm{~d} z \approx N_{n}^{2} z_{b}^{2} J_{0}^{2}\left(m_{n} z_{b}\right)$.

Imposing the approximation $J_{0}(x) \approx \sqrt{\frac{2}{\pi x}} \cos \left(x-\frac{1}{4} \pi\right)$ and the approximate formula of the zero point of $J_{1}\left(x_{n}\right), x_{n} \approx$ $\left(n+\frac{1}{4}\right) \pi$, we get $N_{n} \approx \sqrt{\frac{\pi x_{n}}{2 z_{b}^{2}}}$. Then the normalized KK modes are

$\Psi_{n}(z) \approx \sqrt{\frac{\pi x_{n}}{2 z_{b}^{2}}}\left(\frac{1}{\beta}+|z|\right)^{\frac{1}{2}} J_{0}\left(m_{n}\left(|z|+\frac{1}{\beta}\right)\right)$.

For simplicity, the effective gravitational potential between two point-like sources with mass $M_{1}$ and $M_{2}$ is obtained by the exchange of the graviton zero mode and massive $\mathrm{KK}$ modes, and it can be expressed as [5,69]

$U(r)=-\frac{M_{1} M_{2}}{M_{\mathrm{Pl}}^{2}} \frac{1}{r}-\frac{M_{1} M_{2}}{M_{*}^{3}} \sum_{n=1}^{\infty} \frac{e^{-m_{n} r}}{r}\left|\Psi_{n}(0)\right|^{2}$,

where the four-dimensional Newtonian potential is caused by the zero mode, and the correction term is produced by the massive KK modes. Combining Eqs. (95), (99), and (100), we can get the correction term to the Newtonian potential:

$\Delta U(r)=-\frac{M_{1} M_{2}}{M_{\mathrm{P} 1}^{2}} \sum_{n=1}^{\infty} \frac{\pi x_{n}}{2 z_{b} \beta}\left(2+\beta z_{b}\right) \frac{e^{-x_{n} \frac{r}{z_{b}}}}{r}$.

For $r \gg z_{b}$, the summation term tends to $e^{-x_{n} r / z_{b}} \approx 0$, and the correction can be ignored. For the case of $r \ll z_{b}$, the correction term cannot be ignored and it can be calculated as follows:

$\Delta U(r) \approx-\frac{M_{1} M_{2}}{M_{\mathrm{P} 1}^{2}} \frac{z_{b}^{2}}{r^{3}}$.

It shows that, for $r \ll z_{b}$, the Newtonian potential is corrected by a $1 / r^{3}$ term, which is the leading term. For $r \gg z_{b}$, the correction can be ignored, and we recover the Newtonian potential $U(r) \propto 1 / r$. According to the gravitational experiments of the correction to the Newtonian potential [70-73], we know that the size of the extra dimension, $z_{b}$, should not be larger than the micron scale.

\section{Conclusion and summary}

To summarize, we have investigated the localization and mass spectra of various bulk matter fields on the Weyl brane. We first gave a brief review of the thin brane arising from a five-dimensional Weyl integrable spacetime. Then, we investigated localization of the zero modes for various bulk matter fields (i.e., scalar, vector, and fermion fields) on the brane, and we got the mass spectra of the fields by redefining some dimensionless parameters. We also considered the correction to the four-dimensional Newtonian potential from the massive $\mathrm{KK}$ gravitons.

It was found that the zero modes of various bulk matter fields can be localized on the positive tension brane under some conditions, which are collected in Table 1. It can be seen that the localization conditions for the case of infinite extra dimension are stronger than the case of a finite extra dimension. When the extra dimension is finite, the scalar and vector zero modes can be localized on the positive tension brane even if there is no interaction with the background scalar field (i.e., $\lambda=0$ and $\tau=0$ ).

For the scalar field, we considered two types of couplings with the background scalar field. For the case of the scalardilaton coupling, we found that the mass of the first massive scalar KK mode increases and decreases with the dimensionless coupling constant $\lambda$ and the size of the extra dimension $\bar{z}_{b}$, respectively, and the gap of the mass spectrum decreases with $\bar{z}_{b}$. When the dimensionless size $\bar{z}_{b}>1$, the number of the excited states in a single period, shown in Fig. 2, increases with $\bar{z}_{b}$. When $\bar{z}_{b}<1$, the excited states do not appear in each period, they will emerge after several periods. For the case of the Higgs potential coupling, we fixed the parameter $\bar{\theta}$ and chose the proper value $\bar{u}$, to ensure the zero mode can be localized on the brane. In order to avoid negative $\bar{m}_{n}^{2}$, the mass parameter $\bar{u}$ in the Higgs potential should have an upper limit when the other parameters are fixed.

For the vector field, it was shown that the mass of the first massive vector KK mode increases with the coupling constant $\tau$, and the gap of the mass spectrum decreases with the size of the extra dimension.

For the fermion field, we introduced the usual Yukawa coupling with $F(\omega)=\partial_{z} e^{v \omega}$, and we found that the left- 
chiral fermion zero mode can be localized on the positive tension brane at some conditions. We calculated the mass spectrum of the fermion KK modes for $v=\frac{1}{3}(k+\alpha)$ as an example. The mass of the first excited state increases with the Yukawa coupling constant $\eta$. The size of the extra dimension can also affect the mass gap in the same way as in the cases of the scalar and vector fields. The spectra of various bulk matter fields show the same phenomenon: that the spectrum interval approaches a constant for the higher excited states, while it is relatively sparse for the lower excited states.

For the TT tensor perturbation of the gravitational field, the zero mode is localized on the negative tension brane for the finite extra dimension, and cannot be normalized anymore when the extra dimension is infinite. For the case of the finite extra dimension, the gravity zero mode gives the Newtonian potential, while the gravity massive KK modes will give a correction to the Newtonian potential by a $1 / r^{3}$ term when $r \ll z_{b}$.

Acknowledgements This work was supported by the National Natural Science Foundation of China (Grants Nos. 11522541 and 11375075) and the Fundamental Research Funds for the Central Universities ((Grants No. lzujbky-2016-k04)).

Open Access This article is distributed under the terms of the Creative Commons Attribution 4.0 International License (http://creativecomm ons.org/licenses/by/4.0/), which permits unrestricted use, distribution, and reproduction in any medium, provided you give appropriate credit to the original author(s) and the source, provide a link to the Creative Commons license, and indicate if changes were made. Funded by SCOAP ${ }^{3}$.

\section{References}

1. T. Kaluza, Math. Phys. 1921, 966 (1921)

2. O. Klein. Z. Phys. 37, 895 (1926)

3. V.A. Rubakov, M.E. Shaposhnikov, Phys. Lett. B 125, 136 (1983)

4. L. Randall, R. Sundrum, Phys. Rev. Lett. 83, 3370 (1999). arXiv:hep-ph/9905221

5. L. Randall, R. Sundrum, Phys. Rev. Lett. 83, 4690 (1999). arXiv:hep-th/9906064

6. N. Arkani-Hamed, S. Dimopoulos, G.R. Dvali, Phys. Lett. B 429, 263 (1998). arXiv:hep-ph/9803315

7. B. Bajc, G. Gabadadze, Phys. Lett. B 474, 282 (2000). arXiv:hep-ph/9912232

8. C.-E. Fu, Y.-X. Liu, H. Guo, Phys. Rev. D 84, 044036 (2011). arXiv:1101.0336 [hep-th]

9. H. Hatanaka, M. Sakamoto, M. Tachibana, K. Takenaga, Prog. Theor. Phys. 102, 1213 (1999). arXiv:hep-th/9909076

10. S. Das, D. Maity, S. SenGupta, JHEP 0805, 042 (2008). arXiv:0711.1744 [hep-th]

11. T.R. Slatyer, R.R. Volkas, JHEP 0704, 062 (2007). arXiv:hep-th/0609003

12. S.R. Daemi, M. Shaposhnikov, Phys. Lett. B 492, 361 (2000). arXiv:hep-th/0008079

13. H. Guo, A.H. Aguilar, Y.-X. Liu, D.M. Morejon, R.R.M. Luna, Phys. Rev. D 87, 095011 (2013). arXiv:1103.2430 [hep-th]

14. Y.-X. Liu, H. Guo, C.-E. Fu, J.-R. Ren, JHEP 02, 080 (2010). arXiv:0907.4424 [hep-th]
15. T. Shiromizu, K.I. Maeda, M. Sasaki, Phys. Rev. D 62, 024012 (2000). arXiv:gr-qc/9910076

16. Y.-X. Liu, X.-H. Zhang, L.-D. Zhang, Y.-S. Duan, JHEP 0802, 067 (2008). arXiv:0708.0065 [hep-th]

17. D. Puetzfeld, New Astron. Rev. 49, 59 (2005). arXiv:gr-qc/0404119

18. F.W. Hehl, J.D. McCrea, E.W. Mielke, Y. Neeman, Phys. Rep. 258, 1 (1995). arXiv:gr-qc/9402012

19. R.T. Hammond, Rep. Prog. Phys. 65, 599 (2002)

20. N.B. Cendejas, A.H. Aguilar, Phys. Rev. D 73, 084022 (2006). arXiv:hep-th/0603184

21. H. Cheng, Phys. Rev. Lett. 61, 2182 (1988)

22. D. Hochberg, G. Plunien, Phys. Rev. D 43, 3358 (1991)

23. T. Moon, O. Phillial, J. Sohn, JCAP 11, 005 (2010). arXiv:1002.2549 [hep-th]

24. N.B. Cendejas, A.H. Aguilar, JHEP 0510, 101 (2005). arXiv:hep-th/0511050

25. N.B. Cendejas, A.H. Aguilar, M.A. Reyes, C. Schubert, Phys. Rev. D 77, 126013 (2008). arXiv:0709.3552 [hep-th]

26. Y.-X. Liu, L.-D. Zhang, S.-W. Wei, Y.-S. Duan, JHEP 0808, 041 (2008). arXiv:0803.0098 [hep-th]

27. R.A.C. Correa, D.M. Dantas, P.H.R.S. Moraes, A. de Souza Dutra, C.A.S. Almeida. arXiv:1607.01710 [hep-th]

28. O. Arias, R. Cardenas, I. Quiros, Nucl. Phys. B 643, 187 (2002). arXiv:hep-th/0202130

29. Y.-X. Liu, K. Yang, Y. Zhong, JHEP 1010, 069 (2010). arXiv:0911.0269 [hep-th]

30. A.D. Furlong, A.H. Aguilar, R. Linares, R.R.M. Luna, H.A.M. Tecotl, Gen. Relat. Gravity 46, 1815 (2014). arXiv: 1407.0131 [hepth]

31. I. Oda, Phys. Lett. B 496, 113 (2000). arXiv:hep-th/0006203

32. Y. Sakamura, JHEP 10, 083 (2016). arXiv:1607.07152 [hep-th]

33. Z.-H. Zhao, Q.-Y. Xie, Y. Zhong, Class. Quantum Gravity 32, 035020 (2015). arXiv:1406.3098 [hep-th]

34. G. Alencar, R.R. Landim, M.O. Tahim, R.N. Costa Filho, Phys. Lett. B 739 (2014). arXiv:1409.4396 [hep-th]

35. A.H. Aguilar, A.D. Rojas, E.S. Rodriguez, Eur. Phys. J. C 74, 2770 (2014). arXiv:1401.0999 [hep-th]

36. C.A.V. Araujo, O. Corradini, Eur. Phys. J. C 75, 48 (2015). arXiv: 1406.2892 [hep-th]

37. F.W.V. Costa, J.E.G. Silva, C.A.S. Almeida, Phys. Rev. D 87, 125010 (2013). arXiv:1304.7825 [hep-th]

38. F.W.V. Costa, J.E.G. Silva, D.F.S. Veras, C.A.S. Almeida, Phys. Lett. B 747, 517 (2015). arXiv:1501.00632 [hep-th]

39. T.-T. Sui, L. Zhao. arXiv: 1703.05653 [hep-th]

40. A.E.R. Chumbes, J.M. Hoff da Silva, M.B. Hott, Phys. Rev. D. 85, 085003 (2011). arXiv:1108.3821 [hep-th]

41. W.T. Cruz, A.R.P. Lima, C.A.S. Almeida, Phys. Rev. D. 87, 045018 (2013). arXiv:1211.7355 [hep-th]

42. Z.-H. Zhao, Y.-X. Liu, Y. Zhong, Phys. Rev. D. 90, 045031 (2014). arXiv:1402.6480 [hep-th]

43. G. Alencar, M.O. Tahim, R.R. Landim, C.R. Muniz, R.N. Costa Filho, Phys. Rev. D. 82, 104053 (2010). arXiv:1005.1691 [hep-th]

44. G. Alencar, R.R. Landim, M.O. Tahim, C.R. Muniz, R.N. Costa Filho, Phys. Lett. B. 09, 005 (2010). arXiv:1008.0678 [hep-th]

45. R.C. Fuentevilla, A. Escalante, G. German, A.H. Aguilar, R.R.M. Luna, JCAP 1605, 026 (2016). arXiv: 1412.8710 [hep-th]

46. N.B. Cendejas, D.M. Morejon, R.R.M. Luna, Gen. Relat. Gravity 47, 77 (2015). arXiv:1503.07900 [hep-th]

47. A.E. Bernardini, R. da Rocha, Adv. High Energy Phys. 2016, 3650632 (2016). arXiv:1606.05921 [hep-th]

48. K.P.S. de Brito, R. da Rocha, J. Phys. A 49, 415403 (2016). arXiv:1609.06495 [hep-th]

49. R. Koley, S. Kar, Class. Quantum Gravity 22, 753 (2005). arXiv:hep-th/0407158

50. D. Bazeia, F.A. Brito, R.C. Fonseca, Eur. Phys. J. C 63, 163 (2009). arXiv:0809.3048 [hep-th] 
51. A. Melfo, N. Pantoja, J.D. Tempo, Phys. Rev. D 73, 044033 (2006). arXiv:hep-th/0601161

52. C.A.S. Almeida, R. Casana, M.M. Ferreira, A.R. Gomes, Phys. Rev. D 79, 125022 (2009). arXiv:0901.3543 [hep-th]

53. R. Davies, D.P. George, Phys. Rev. D 76, 104010 (2007). arXiv:0705.1391 [hep-th]

54. L.B. Castro, Phys. Rev. D 83, 045002 (2011). arXiv:1008.3665 [hep-th]

55. L.B. Castro, L.E.A. Meza, Europhys. Lett 102, 21001 (2013). arXiv:1011.5872 [hep-th]

56. Y.-X. Liu, Z.-G. Xu, F.-W. Chen, S.-W. Wei, Phys. Rev. D 89, 086001 (2014). arXiv:1312.4145 [hep-th]

57. Y.-Y. Li, Y.-P. Zhang, W.-D. Guo, Y.-X. Liu. arXiv:1701.02429 [hep-th]

58. C. Csaki, M. Graesser, C. Kolda, J. Terning, Phys. Lett. B 462, 34 (1999). arXiv:hep-th/9906513

59. J.M. Cline, C. Grojean, G. Servant, Phys. Rev. Lett. 83, 4245 (1999). arXiv:hep-th/9906523

60. T. Shiromizu, K. Maeda, M. Sasaki, Phys. Rev. D 62, 024012 (2000). arXiv:hep-th/9910076

61. K. Yang, Y. Zhong, Y.-X. Liu, S.-W. Wei. Int. J. Mod. Phys. A 29, 1450120 (2014). arXiv:1108.5436 [hep-th]

62. V. Dzhunushaliev, V. Folomeev, M. Minamitsuji, Rep. Prog. Phys. 73, 066901 (2010). arXiv:0904.1775 [gr-qc]
63. V. Dzhunushaliev, V. Folomeev, B. Kleihaus, J. Kunz, JHEP 1004, 130 (2010). arXiv:0912.2812 [gr-qc]

64. D. Bazeia, A.S. Lobao, R. Menezes, Phys. Lett. B 743, 98 (2015). arXiv:1502.04757 [hep-th]

65. M. Peyravi, N. Riazi, F.S.N. Lobo, Eur. Phys. J. C 76, 247 (2016). arXiv: 1504.04603 [gr-qc]

66. T. Gherghetta, A Holographic View of Beyond the Standard Model Physics. arXiv:1008.2570 [hep-ph]

67. O. DeWolfe, D.Z. Freedman, S.S. Gubser, A. Karch, Phys. Rev. D 62, 046008 (2000). arXiv:hep-th/9909134

68. B. Guo, Y.-X. Liu, K. Yang, X.-H. Meng. arXiv:1501.02674 [hepth]

69. C. Csaba, J. Erlich, T.J. Hollowood, Y. Shirman, Nucl. Phys. B 581, 309 (2000). arXiv:hep-th/0001033

70. M. Eingorn, A. Kudinova, A. Zhuk, Gen. Relat. Gravity 44, 2257 (2012). arXiv:1111.4046 [gr-qc]

71. L. Iorio, Ann. Phys. 524, 371 (2012). arXiv:1112.3517 [gr-qc]

72. R. Linares, H.A.M. Tecotl, O. Pedraza, L.O. Pimentel, Phys. Rev. D 89, 066002 (2014). arXiv:1312.7060 [hep-ph]

73. W.H. Tan, S.Q. Yang, C.G. Shao, J. Li, A.B. Du, B.F. Zhan, Q.L. Wang, P.S. Luo, L.C. Tu, J. Luo, Phys. Rev. Lett. 116, 131101 (2016) 